\title{
Risk factors of acute and overuse musculoskeletal injuries among young conscripts: a population-based cohort study
}

Henri Taanila ${ }^{1,2,3^{*}}$, Jaana H Suni², Pekka Kannus ${ }^{2,3}$, Harri Pihlajamäki ${ }^{4,5}$, Juha-Petri Ruohola', Jarmo Viskari ${ }^{6}$ and Jari Parkkari ${ }^{2,3}$

\begin{abstract}
Background: Military service in Finland is compulsory for all male citizens and annually about $80 \%$ of 19-year-old men enter into the service. The elevated risk for many chronic diseases and loss of function among those who are inactive and unfit can be often detected already in youth. On the other hand, activity-induced injuries among young are true public health issue. The purpose of the present prospective cohort follow-up study was to evaluate predictive associations between acute or overuse injuries and their various intrinsic risk factors.
\end{abstract}

Methods: Four successive cohorts of conscripts who formed a representative sample of Finnish young men were followed for 6 months. At the beginning of the service, the risk factors of injuries were measured and recorded and then the acute and overuse injuries treated at the garrison clinic were identified. Predictive associations between injuries and their risk factors were examined by multivariate Cox's proportional hazard models.

Results: Of the 1411 participants, 27\% sustained an acute injury and 51\% suffered from overuse injury. Concerning acute injuries, highest risk for severe injuries were detected among conscripts with low fitness level in both the standing long-jump and push-up tests (hazard rate, $\mathrm{HR}=5.9 ; 95 \% \mathrm{Cl}$ : 1.6-21.3). A history of good degree in school sports was not a protective factor against acute injuries. High waist circumference and, on the other hand, being underweight according to BMl increased the HR for overuse injuries. Brisk leisure time physical activity before military entry was a protective factor against overuse injuries. Poor result in Cooper's test was a warning signal of elevated risk of overuse injuries.

Conclusion: We confirmed previous findings that low level of physical fitness is predictor for musculoskeletal injuries during intensive physical training. The U-shaped relationship between body composition and overuse injuries was noticed indicating that both obesity and underweight are risk factors for overuse injuries. Persons with excellent sports skills according to their earlier degrees in school sports had similar HR for acute injuries than those with poorer degrees. This indicates that school-age sports skills and fitness do not carry far and therefore preventive programmes are needed to prevent activity-induced injuries.

Keywords: Risk factors, Sporting injuries, Physical fitness, Physical activity, Military training

\footnotetext{
* Correspondence: taanila.henri.p@student.uta.fi

${ }^{1}$ Research Department, Centre for Military Medicine, Lahti and Helsinki,

Finland

${ }^{2}$ Tampere Research Centre of Sports Medicine, and Injury and Osteoporosis

Research Center, UKK Institute, PO Box 30FIN-33501 Tampere, Finland

Full list of author information is available at the end of the article
} 


\section{Background}

Numerous well-conducted studies have shown that the least active and unfit are at greatest risk for a variety of chronic diseases, loss of function, and all-cause mortality $[1,2]$. Western lifestyle is becoming increasingly sedentary [3], which is associated with a range of poor health outcomes, typically high levels of obesity, type 2 diabetes, cardiovascular problems [4] and mortality [5]. Moreover, there is clear evidence that low physical fitness rather than just low levels of physical activity (PA) or sedentary lifestyle, is an independent risk factor for chronic diseases, poor health outcomes [6-8] and musculoskeletal injuries $[2,9,10]$. The other side of the medal is that the risk of musculoskeletal injury increases with an increase in PA. In fact, the increasing number of activity-induced injuries among adolescents and young adults is becoming a true public health burden [11-13].

In military environment, previous physical inactivity [14-16] and low aerobic endurance [14,17-22] have been shown to be associated with musculoskeletal injuries during military training. Musculoskeletal injuries are the main reason for morbidity in military populations [23,24]. Moreover, training-related injuries are the main reason for disability, long-term rehabilitation, functional impairment, and premature discharges from military service [23,25-27]. In the Finnish Defence Forces, musculoskeletal injuries and disorders are the second highest reason for premature discharge from military service, and their number has been increasing [28]. Given that $80 \%$ of young men in Finland enter into military service, the high number of musculoskeletal injuries affects public health [24].

The purpose of the present 6-month prospective follow-up study of four successive cohorts conscripted in the Finnish army was to evaluate predictive associations between acute injuries (AIs) or overuse injuries (OIs) and their various intrinsic risk factors, including socio-economic, health, health behaviour, and physical fitness variables. We hypothesised that low levels of physical fitness, sedentary lifestyle and health damaging behaviour at the beginning of military service are associated with an increased incidence of acute and especially overuse injuries among young men during intensive physical training.

\section{Methods}

\section{Study design}

In Finland, military service or alternative civil service is compulsory for all male citizens over 18 years of age and voluntary for women. Annually about $80 \%$ of 19 -year-old men enter into the service. The service period varies from 6 to 12 months. The study was carried out in the Pori Brigade, a typical Finnish garrison. Companies without special qualification requirements were enrolled in the study including anti-tank, signal, mortar, and engineer companies forming a representative sample of conscripts $(N=1513)$. During the study period, four cohorts of conscripts started service in the brigade (Figure 1). Annually, the conscripts of each age-cohort were randomly assigned into the study companies. The above-described study sample was the same as in our previous study reporting predictors of military discharge [29]. Approval for the study protocol was obtained from the Ethics Committee of Pirkanmaa Hospital District (ETL-code R07076).

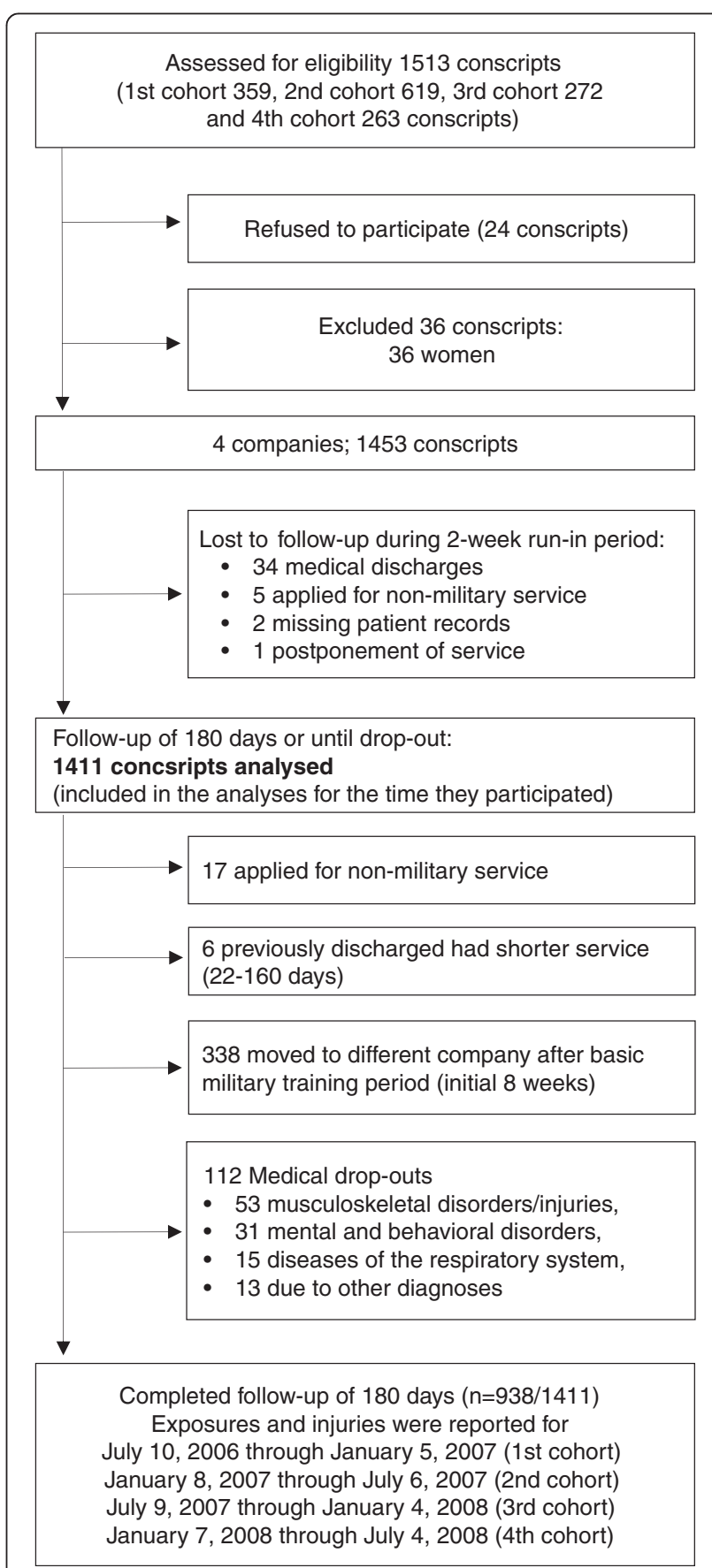

Figure 1 Flow of participants through the study. 


\section{Participants}

The rate of written informed consent to participate was high (98\%). Because there were only 36 women in the study (2.4\%), their data were excluded from the analysis. Conscripts entering military service were young healthy men, all of whom had a medical check-up by a clinician during the 12 months before entering into the military. The health status of the conscripts was rechecked at baseline using routine medical screenings performed by a physician. To exclude injuries and illnesses originating before the onset of military service, conscripts discharged from the service at the medical screenings during the 2 -week run-in period were excluded from the analyses leaving 1411 conscripts in the analyses. All subjects were planned to be followed for 180 days beginning on the first day of service, but some drop-out from the military or changed company and this was taken into account when calculating exposure times (Figure 1).

\section{Baseline characteristics of participants}

All conscripts filled in a standard pre-information questionnaire during the first week of military service. Questions charted conscripts' socio-economic factors, health, and health behaviour at the baseline of the study. Baseline characteristics of the study participants are presented in Table 1. Assessment of physical fitness was conducted in $97 \%$ of the conscripts during the first 2 weeks of their service. The assessment of physical fitness and body composition were applied according to standard procedures in the Finnish Defence Forces and are reported in the previous articles $[17,29]$ (Figures 2, 3, 4, 5 and 6; informed consent to publish was obtained from the individual in the figures). Because excellent results in the 12-min running test were uncommon $(<4 \%)$, the two highest levels, good and excellent, were combined to obtain a group of equal size for comparison. Individual physical fitness test results were combined into a single variable to explore whether coimpairment in aerobic and muscular fitness would increase the HR for injuries. Co-impairment was defined as a poor result in both measured fitness tests according to the standard result categories [17,30] (Figure 2, 3, 4, 5 and 6).

\section{Physical training programme}

Conscripts performed 8 weeks of routine basic physical training programme with a gradual increase in intensity. There was an average of 17 hours of military actions per week mostly at low-to-moderate intensity including marching, cycling, skiing, orienteering, swimming, drill training and combat training. The 2-month basic training period was followed by a specific military training programme depending on the company and service duration. During this 4-month period of service, the volume and intensity of physical training was maintained at approximately the same level in different companies. Military tasks practiced in the four companies were partly different after the 2-month basic training period due to different soldiery assignment

Table 1 Baseline characteristics of 1411 male conscripts by company

\begin{tabular}{|c|c|c|c|c|c|c|}
\hline Variable & Anti-tank & Signal & Mortar & Engineer & Missing & P-value $^{1}$ \\
\hline Number of conscripts & 263 & 540 & 363 & 245 & $0(0 \%)$ & - \\
\hline Age, median, years & 19 & 19 & 19 & 19 & $0(0 \%)$ & $0.422^{2}$ \\
\hline Body mass index, median, $\mathrm{kg} / \mathrm{m}^{2}$ & 23.4 & 22.6 & 23.3 & 23.6 & $139(10 \%)$ & $0.003^{2}$ \\
\hline Waist circumference, median, cm & 87.0 & 84.9 & 85.6 & 87.0 & $101(7 \%)$ & $0.001^{2}$ \\
\hline 12-minute run test result, median, $\mathrm{m}$ & 2310 & 2308 & 2500 & 2400 & $42(3 \%)$ & $<0.001^{2}$ \\
\hline Conscript's physical fitness index $(\mathrm{CPFI})^{4}$, median, points & 15.05 & 14.75 & 17.00 & 15.50 & $46(3 \%)$ & $<0.001^{2}$ \\
\hline Hometown population $\geq 10,000$ persons, $\%$ & $59 \%$ & $66 \%$ & $59 \%$ & $57 \%$ & $24(2 \%)$ & $0.044^{3}$ \\
\hline High level of education ${ }^{5}, \%$ & $48 \%$ & $38 \%$ & $49 \%$ & $35 \%$ & $22(2 \%)$ & $<0.001^{3}$ \\
\hline High level of previous physical activity ${ }^{6}, \%$ & $31 \%$ & $24 \%$ & $42 \%$ & $36 \%$ & $23(2 \%)$ & $<0.001^{3}$ \\
\hline Good self-assessed health ${ }^{7}, \%$ & $57 \%$ & $47 \%$ & $61 \%$ & $51 \%$ & $22(2 \%)$ & $<0.001^{3}$ \\
\hline Chronic impairment or disability, $\%$ & $17 \%$ & $15 \%$ & $16 \%$ & $17 \%$ & $27(2 \%)$ & $0.802^{3}$ \\
\hline Clear musculoskeletal symptoms ${ }^{8}, \%$ & $28 \%$ & $32 \%$ & $26 \%$ & $27 \%$ & $23(2 \%)$ & $0.339^{3}$ \\
\hline Previous or current regular smoker, \% & $43 \%$ & $47 \%$ & $44 \%$ & $57 \%$ & $26(2 \%)$ & $0.004^{3}$ \\
\hline Use of alcohol $\geq 3$ times per week, $\%$ & $16 \%$ & $19 \%$ & $15 \%$ & $20 \%$ & $23(2 \%)$ & $0.318^{3}$ \\
\hline
\end{tabular}

${ }^{1} \mathrm{P}$-value for difference between the companies.

${ }^{2} \mathrm{P}$-value was calculated using a Kruskal-Wallis test for median difference.

${ }^{3} \mathrm{P}$-value was calculated using $\mathrm{X} 2$ statistics for difference.

${ }^{4} \mathrm{CPFI}=(12$-min running test result (metres) $+100 \times$ muscle fitness test points) $/ 200$, [Excellent $(\mathrm{CPFI} \geq 21.00), \mathrm{Good}(17.00 \leq \mathrm{CPFI}<21.00)$, Fair good $(13.00 \leq \mathrm{CPFI}$

$<17.00)$, Poor $(\mathrm{CPFI}<13.00)$.

${ }^{5}$ Graduated or studies in higher education institution.

${ }^{6}$ Sweating exercise at least three times per week during the last month before entering the military.

${ }^{7}$ Compared to age-mates.

${ }^{8}$ Symptoms lasting more than 7 days in at least one anatomical region during the last month before entering the military. 


\begin{abstract}
PUSH-UP TEST
Purpose: To assess dynamic strength of the upper body and the ability to stabilise the trunk.

Method: The conscript starts from the lowest face-down position and hands are kept shoulder-wide level. During the push-up, a conscript was first required to fully extend his arms while keeping the body straight with tensed trunk muscles. In the second phase, the body was lowered to the down position with an elbow angle of $90^{\circ}$.

Outcome: Number of consecutive repeats completed in 60 seconds. Result categories: Excellent ( $\geq 38)$, Good $(\geq 30)$, Fair good $(\geq 22)$ and Poor $(<22)$ [repeats].
\end{abstract}

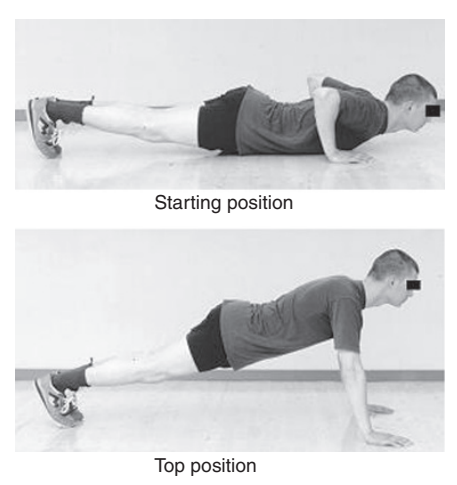

Figure 2 Description of push-up test.

objectives depending of the company. After this, however, the core of the military physical training was still based on the same military actions as in the beginning of the service.

\section{Injury definition and data collection}

Injury was defined as an event that resulted in physical damage or pain for which the conscript sought medical care from the garrison clinic. During military service, all conscripts had to use the services of the military healthcare units. The data were collected from July 10, 2006 to July 4, 2008 (Figure 1). The date, anatomical location, etiological circumstances, severity and diagnosis were registered in electronic patient records. Injuries that occurred during the conscript's leisure time or on the way to vacation or back to garrison were also included in the analyses. Because the conscripts may have sought medical care several times due to the same event, the total number of health clinic visits exceeded the number of injuries. The health clinic visits were considered to be for the same injury when the conscript had sustained an injury of the same type and location during the preceding 2 weeks or if a physician had marked on the patient files that the reason for the visit was related to the previous injury.

The type of injury was categorised as acute if it had a sudden onset involving known trauma [31-33]. For example, sprains, strains, ligament ruptures, and joint dislocations were categorised as acute. Overuse-related injuries had a gradual onset without known trauma $[31,33]$ and they were described as a pain syndrome of the musculoskeletal system, where symptoms appeared during physical activities at previously symptomless body part [34]. One of the study physicians (HT) checked all the patient records in order to differentiate acute injury (AI) from overuse injury (OI).

Injury severity was categorised according to the number of days of limited duty. Severe injury was defined as an incidence of time loss of at least 7 active service days. Limited duty involved a physical restriction that prevented the conscript from fully participating in military training events. Discharge from military service was indicated when a physician determined a conscript unable to continue military training. Discharges from military service due to musculoskeletal injuries were registered as severe injuries. Specific details of these discharges including risk factor analyses were presented previously [29].

\section{Statistical analysis}

IBM SPSS Statistics 22.0 for Windows software was used for statistical analysis. AI and OI incidences were calculated

\section{STANDING LONG-JUMP TEST}

Purpose: To measure explosive force production of the lower limb extensor muscles as well as motor control.

Method: The jump starts with legs close to each other and bilateral takeoff is assisted by swinging of the upper body and arms. The landing is bilateral and shortest distance expressed in metres from the landing to the starting point was measured.

Outcome: The conscript has two attempts and the best result is registered. Result categories: Excellent $(\geq 2,40$ $m)$, Good $(\geq 2,20 \mathrm{~m})$, Fair good $(\geq 2,00 \mathrm{~m})$ and Poor $(<2,00 \mathrm{~m})$.

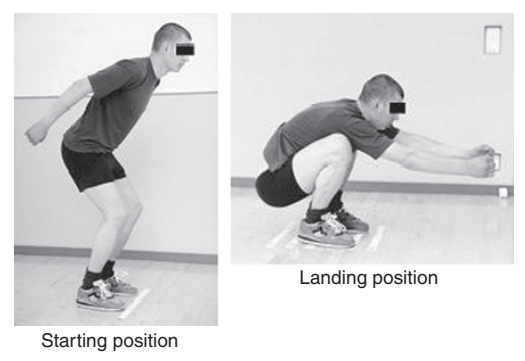

Figure 3 Description of standing long jump test. 


\section{SIT-UP TEST}

Purpose: To measure dynamic endurance of abdominal and hip-flexor muscles.

Method: The conscript is lying on the floor supine with hands behind the neck. The knees are flexed at an angle of $90^{\circ}$, and an assistant supports the ankles (contrary to the picture). The conscript raises upper body until his elbows touches the knees and then returns to the starting position where both scapulas touches the floor.

Outcome: Number of consecutive repeats completed in 60 seconds. Result categories: Excellent $(\geq 48)$, Good ( $\geq 40)$, Fair good $(\geq 32)$ and Poor $(<32)$ [repeats].

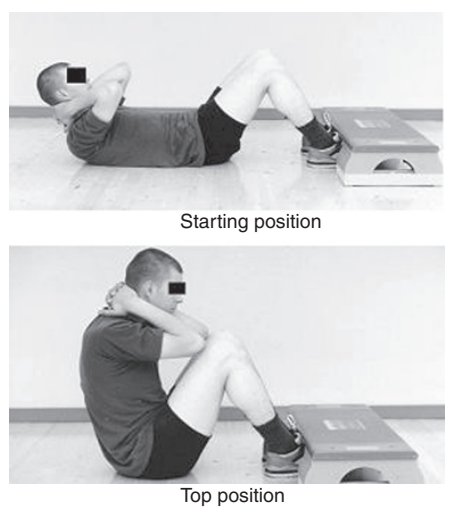

Figure 4 Description of sit-up test.

by dividing the number of conscripts with one or more AIs or OIs treated in the garrison clinic (numerator) by the total number of conscripts (denominator) and expressed as a percentage. The incidence rates were calculated by dividing the number of injured conscripts by the exposure time and expressed per 1000 person-days. Exposure times for AI and $\mathrm{OI}$ incidence rates were calculated until onset of the conscript's first AI or OI, respectively.

Cox's proportional hazard models were applied to study the prospective associations between baseline characteristics and outcome. The primary outcomes were defined as an incidence of any AI or OI. The secondary outcomes were defined as an incidence of time loss of at least 7 active service days due to one or several acute (referred to as a severe AI) or overuse (referred to as a severe OI) injuries to explore predictors of injuries that debilitate service clearly. In the first phase of the Cox regression, each independent variable was analysed one at a time (univariate). Results are expressed as hazard ratios (HR) and calculated with 95\% CIs with age at baseline forced into the model.

A multivariate Cox's proportional hazard models (entermethod) was used to identify independent risk factors for $\mathrm{AI}$ and $\mathrm{OI}$ incidence and examine interactions between risk factors. Stepwise procedures were not used. Presenting the univariate HRs parallel to adjusted HRs was made to allow the reader to perceive how adjustments affect studied risk factors. Cox's proportional hazard model assumptions were assessed by using the Schoenfeld residuals [35]. Conceptually compatible and logical risk factors that were possibly significant variables $(P<0.20)$ in the initial univariate models were included in the multivariate models. To exclude colinearity, we identified the variables that were highly correlated $(r>0.5)$, and we included in the multivariate model only those variables that had a higher level of significance (e.g. waist circumference was selected in the multivariate model and BMI was not selected). Considering AIs and OIs, the included adjusting variables for multivariate models are presented in footnote of the Table 2 and Table 3, respectively. A $P$ value of less than 0.05 was considered statistically significant when interpreting the results from Cox's proportional hazard models.

\section{Results}

During the 6-month follow-up of four successive cohorts, there were 550 acute injuries (AIs) and 1351 overuse injuries (OIs) in 1411 persons. These injuries accounted for 3435 garrison health clinic visits. Of the 1411 participants,

\section{BACK LIFT TEST}

Purpose: To measure dynamic endurance of back and hipextensor muscles.

Method: The conscript lies prone on the floor with hands behind the neck in the starting position. An assistant supports the legs (contrary to the picture). During the movement, the upper body is lifted until the scapulas are approximately $30 \mathrm{~cm}$ higher than in the starting point. Thereafter, the upper body is lowered down back to the starting position.

Outcome: Number of consecutive repeats completed in 60 seconds. Result categories: Excellent $(\geq 60)$, Good $(\geq 50)$, Fair good $(\geq 40)$ and Poor $(<40)$ [repeats].

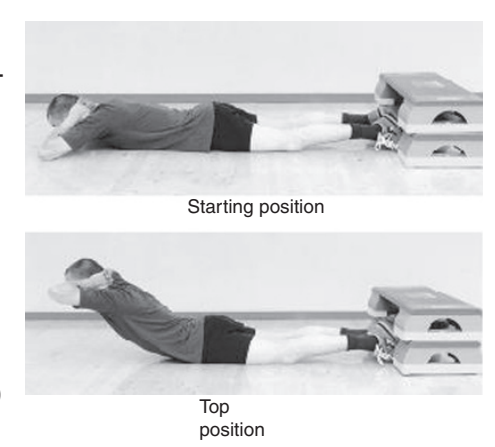

Figure 5 Description of back lift test. 


\section{PULL-UP TEST}

Purpose: To measure dynamic endurance of flexor muscles in arm and shoulder.

Method: The conscript is required to raise his chin over a bar and then return to the starting point with elbows fully extended.

Outcome: Repeats without time limit. Repeats have to be consecutive and intermission in the starting point is not allowed. Result categories: Excellent $(\geq 14)$, Good $(\geq 10)$, Fair good $(\geq 6)$ and Poor $(<6)$ [repeats].
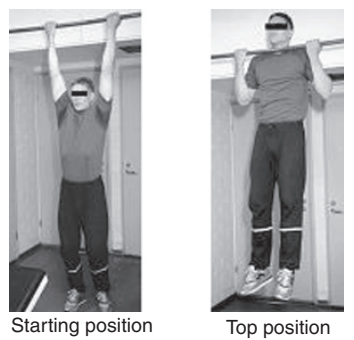

Figure 6 Description of pull-up test.

27\% ( $\mathrm{n}=386)$ sustained an AI and 51\% ( $\mathrm{n}=721)$ suffered from OI during the 6-month service. Considering severe injuries, a total of 89 (6\%) conscripts suffered from severe AI, and 251 (18\%) conscripts sustained a severe OI ( $\geq 7$ service days lost due to injuries). The incidence rate for AI was 2.31 (95\% CI: 2.09-2.55) and for OI 5.37 (95\% CI: 5.00-5.78) per 1000 person-days, respectively. The AI or OI incidences for the first (AI: 29\%; OI: $51 \%$ ), second (AI: 28\%; OI: 52\%), third (AI: 25\%; OI: 53\%), and fourth (AI: 27\%; OI: 48\%) cohorts did not vary significantly $(P>0.20)$. In addition, the AI or OI incidences were not significantly different $(P>0.20)$ among arrivals entering the military in July (AI: 27\%; OI: $52 \%$ ) and in January (AI 27\%; OI: 50\%).

OIs (71\%) were more than twice as prevalent as AIs (29\%). Most injuries were in the lower extremities (67\%) followed by the back (18\%), upper extremities including shoulders $(10 \%)$, head $(2 \%)$ and other parts of the body (torso excluding back; 3\%).

\section{Injury severity and associated activities}

The majority (67\%) of AIs were classified as minimal, leading to a maximum 3-day exemption from military training, while $18 \%$ of AIs accounted for 4-7 days , 11\% for $8-28$ days and $3 \%$ for over 28 days exemption from military training. Corresponding figures for OIs were $65 \%$ for a maximum 3-day exemption from military training, 20\% 4-7 days, $11 \%$ for $8-28$ days and $4 \%$ for over 28 days exemption from military training. Some injuries (6\%) occurred during vacations and three cases $(0.2 \%)$ occurred while the conscript was traveling to vacation or back to the garrison. Fifty-three (3.8\%) conscripts were discharged from military service due to musculoskeletal injuries after the 2-week run-in period. Mostly (76\%), these discharges were due to overuse musculoskeletal conditions (Figure 1).

\section{Risk factors of acute injuries}

With regard to AIs, Tables 2 and 4 show the distribution and the hazard ratios of most important socioeconomic, health, health behaviour (Table 2) and physical fitness variables (Table 4) in the age-adjusted and multivariate models.
From the socioeconomic background variables, a conscript's poor school success (educational level and degrees combined) was the strongest risk factor. In multivariate analyses, poor school success was associated clearly with $\mathrm{AI}$ and severe $\mathrm{AI}(\mathrm{HR}=5.3$; 95\% CI: 1.9-15.1) compared to excellent school success in a graded manner. Living in bigger city with over 90000 inhabitants was associated with AI. In addition, company was associated with AI, HR being lowest in the anti-tank company and highest in the engineer company (Table 2).

With regard to health, pre-service musculoskeletal symptoms were associated with incidence of $\mathrm{AI}$ and severe $\mathrm{AI}$ in the age-adjusted model. After further adjustments, former sports injury ( $\mathrm{HR}=2.0 ; 95 \% \mathrm{CI}$ : 1.1-3.6) remained predictive of severe AI. High BMI and high waist circumference increased the HR for severe AI in the age-adjusted model, but were not significant in multivariate model (Table 2).

With regard to health behaviours, health damaging behaviour was associated with incidence of AI and severe AI. Smoking and frequency of drunkenness were associated with AI in the age-adjusted model, but after final adjustments, the associations weakened (Table 2). High pre-service alcohol intake was associated with severe AI also after further adjustments in multivariate model (HR $=2.0 ; 95 \% \mathrm{CI}: 1.2-3.2)$. A history of good or excellent degree in school sports was not a protective factor for AI (Table 2).

With regard to physical fitness single test items of poor fitness in standing long-jump and push-up test showed predictive associations with incidence AI or severe AI. Results of pull-up, back lift or sit-up tests were not associated with AIs. Association between poor push-up test and AI incidence, however, diminished after multivariable adjustments. Highest HR for both AI and severe AI were detected among conscripts with low fitness level in both standing long-jump and pushup tests $(\mathrm{HR}=5.9 ; 95 \% \mathrm{CI}: 1.6-21.3)$ or in standing long-jump and Cooper's 12-min running test (Table 4).

\section{Risk factors of overuse injuries}

Considering OIs, Tables 3 and 5 show the distribution and the hazard ratios of most important socioeconomic, health, 
Table 2 Hazard ratios (HR) for any acute injury (AI) and severe acute injury (severe AI) incidence by most important socioeconomic, health and health behaviour variables at baseline

\begin{tabular}{|c|c|c|c|c|c|c|}
\hline Baseline variable & Category & $\begin{array}{l}\text { Total number } \\
\text { (\% of experienced Al; } \\
\% \text { of experienced } \\
\text { severe Al) }\end{array}$ & $\begin{array}{l}\text { HR for Al incidence } \\
(\mathrm{n}=386)^{*}\end{array}$ & $\begin{array}{l}\text { HR for Al incidence } \\
(\mathrm{n}=354)^{* *}\end{array}$ & $\begin{array}{l}\text { HR for severe Al } \\
\text { incidence }(\geq 7 \text { service } \\
\text { days lost) }(\mathrm{n}=89)^{*}\end{array}$ & $\begin{array}{l}\text { HR for severe } A \\
\text { incidence }(\geq 7 \mathrm{~s} \\
\text { days lost) }(\mathrm{n}=\varepsilon\end{array}$ \\
\hline \multicolumn{7}{|l|}{ Socioeconomic variables } \\
\hline \multirow[t]{2}{*}{ Age } & 18-19 years & $1052(27 ; 6)$ & 1 (Referent) & 1 (Referent) & 1 (Referent) & 1 (Referent) \\
\hline & 20-28 years & $359(27 ; 6)$ & $1.1(0.8-1.3)$ & $1.0(0.8-1.3)$ & $1.1(0.7-1.7)$ & $1.1(0.7-1.8)$ \\
\hline \multirow[t]{3}{*}{ Father's occupation } & Not physical & $488(24 ; 6)$ & 1 (Referent) & 1 (Referent) & 1 (Referent) & 1 (Referent) \\
\hline & Physical & $590(29 ; 7)$ & $1.3(1.0-1.6)$ & $1.2(1.0-1.6)$ & $1.0(0.6-1.7)$ & $1.0(0.6-1.6)$ \\
\hline & Unclear or unemployed & $302(28 ; 6)$ & $1.2(0.9-1.6)$ & $1.1(0.8-1.4)$ & $0.9(0.5-1.6)$ & $0.8(0.4-1.5)$ \\
\hline \multirow{4}{*}{$\begin{array}{l}\text { School success (educational level } \\
\text { and grades combined) }\end{array}$} & Excellent $^{1}$ & $218(20 ; 4)$ & 1 (Referent) & 1 (Referent) & 1 (Referent) & 1 (Referent) \\
\hline & Good $^{2}$ & $608(28 ; 5)$ & $1.5(1.1-2.1)$ & $1.5(1.1-2.1)$ & $1.4(0.6-3.0)$ & $1.5(0.6-3.7)$ \\
\hline & Satisfactory $^{3}$ & $467(27 ; 8)$ & $1.5(1.1-2.1)$ & $1.4(0.9-2.0)$ & $2.4(1.1-5.2)$ & $2.5(1.0-6.3)$ \\
\hline & Poor $^{4}$ & $96(35 ; 13)$ & $2.5(1.6-3.9)$ & $2.4(1.5-3.9)$ & $4.5(1.8-11.1)$ & $5.3(1.9-15.1)$ \\
\hline \multirow{3}{*}{$\begin{array}{l}\text { Urbanisation level of the } \\
\text { place of residence }\end{array}$} & Countryside & $227(23 ; 5)$ & 1 (Referent) & 1 (Referent) & 1 (Referent) & 1 (Referent) \\
\hline & Town, $<10000$ inhabitants & $310(25 ; 8)$ & $1.1(0.8-1.6)$ & $1.0(0.7-1.4)$ & $1.5(0.7-3.0)$ & $1.3(0.6-2.5)$ \\
\hline & City, $\geq 90000$ inhabitants & $298(33 ; 8)$ & $1.5(1.1-2.1)$ & $1.5(1.1-2.2)$ & $1.4(0.7-2.9)$ & $1.5(0.7-3.0)$ \\
\hline \multirow[t]{4}{*}{ Company } & Anti-tank company & $263(24 ; 6)$ & 1 (Referent) & 1 (Referent) & 1 (Referent) & 1 (Referent) \\
\hline & Signal company & $540(27 ; 6)$ & $1.3(0.9-1.7)$ & $1.2(0.9-1.7)$ & $0.9(0.5-1.7)$ & $1.0(0.6-2.0)$ \\
\hline & Mortar company & $363(21 ; 5)$ & $1.2(0.9-1.7)$ & $1.2(0.8-1.7)$ & $1.2(0.6-2.2)$ & $1.4(0.7-3.0)$ \\
\hline & Engineer company & $245(40 ; 9)$ & $1.9(1.4-2.6)$ & $1.9(1.3-2.6)$ & $1.5(0.8-2.9)$ & $1.6(0.8-3.2)$ \\
\hline \multicolumn{7}{|l|}{ Health variables } \\
\hline \multirow[t]{4}{*}{ Body mass index ${ }^{5}\left(\mathrm{BMI}=(\mathrm{kg}) /(\mathrm{m})^{2}\right)$} & Underweight (<18.5) & $56(21 ; 2)$ & $0.9(0.5-1.5)$ & $0.9(0.5-1.6)$ & $0.3(0.1-2.4)$ & $0.3(0.1-2.3)$ \\
\hline & Normal $(18.5-25.0)$ & $812(26 ; 6)$ & 1 (Referent) & 1 (Referent) & 1 (Referent) & 1 (Referent) \\
\hline & Pre-obese $(25.0-30.0)$ & $300(29 ; 7)$ & $1.1(0.9-1.4)$ & $1.1(0.8-1.4)$ & $1.2(0.7-2.0)$ & $1.1(0.6-1.8)$ \\
\hline & Obese $(\geq 30.0)$ & $104(40 ; 12)$ & $1.7(1.2-2.3)$ & $1.3(0.9-1.9)$ & $2.0(1.1-3.8)$ & $1.2(0.6-2.4)$ \\
\hline \multirow[t]{3}{*}{ Waist circumference $(\mathrm{WC}, \mathrm{cm})$} & Thin $(<80 \mathrm{~cm})$ & $271(25 ; 4)$ & $1.0(0.8-1.3)$ & $1.0(0.8-1.4)$ & $0.8(0.4-1.6)$ & $0.9(0.4-1.6)$ \\
\hline & Average $(80-93.5 \mathrm{~cm})$ & $739(27 ; 6)$ & 1 (Referent) & 1 (Referent) & 1 (Referent) & 1 (Referent) \\
\hline & Increased $(94-101.5$ cm) & $178(32 ; 7)$ & $1.2(0.9-1.7)$ & $1.1(0.8-1.5)$ & $1.3(0.7-2.4)$ & $1.0(0.5-1.8)$ \\
\hline \multirow{2}{*}{$\begin{array}{l}\text { Sum factor of musculoskeletal } \\
\text { symptoms }\end{array}$} & Mild symptoms ${ }^{7}$ & $548(28 ; 6)$ & $1.2(0.9-1.5)$ & $1.2(0.9-1.5)$ & $1.2(0.7-2.0)$ & $1.0(0.6-1.7)$ \\
\hline & Clear symptoms ${ }^{8}$ & $400(30 ; 8)$ & $1.4(1.0-1.8)$ & $1.2(0.9-1.6)$ & $1.8(1.0-3.0)$ & $1.3(0.8-2.3)$ \\
\hline
\end{tabular}


Table 2 Hazard ratios (HR) for any acute injury (AI) and severe acute injury (severe Al) incidence by most important socioeconomic, health and health behaviour variables at baseline (Continued)

\begin{tabular}{|c|c|c|c|c|c|c|}
\hline \multirow[t]{2}{*}{ Chronic disease } & No & $1012(28 ; 6)$ & 1 (Referent) & 1 (Referent) & 1 (Referent) & 1 (Referent) \\
\hline & Yes & $377(26 ; 7)$ & $1.0(0.8-1.2)$ & $1.0(0.8-1.2)$ & $1.1(0.7-1.8)$ & $1.1(0.7-1.8)$ \\
\hline \multirow[t]{2}{*}{ Sports injury during last month } & No & $1254(27 ; 6)$ & 1 (Referent) & 1 (Referent) & 1 (Referent) & 1 (Referent) \\
\hline & Yes & $130(29 ; 10)$ & $1.2(0.8-1.6)$ & $1.2(0.8-1.6)$ & $1.8(1.0-3.3)$ & $2.0(1.1-3.6)$ \\
\hline \multicolumn{7}{|l|}{ Health behaviour variables } \\
\hline \multirow[t]{2}{*}{ Smoking habits } & Never smoked regularly & $735(25 ; 5)$ & 1 (Referent) & 1 (Referent) & 1 (Referent) & 1 (Referent) \\
\hline & Has smoked regularly & $650(30 ; 8)$ & $1.3(1.1-1.6)$ & $1.2(0.9-1.6)$ & $1.6(1.0-2.4)$ & $1.2(0.7-1.9)$ \\
\hline \multirow[t]{2}{*}{ Use of alcohol ${ }^{9}$} & $<3$ times per week & $1148(26 ; 5)$ & 1 (Referent) & 1 (Referent) & 1 (Referent) & 1 (Referent) \\
\hline & $\geq 3$ times per week & $240(33 ; 12)$ & $1.3(1.0-1.6)$ & $1.1(0.8-1.4)$ & $2.3(1.5-3.6)$ & $2.0(1.2-3.2)$ \\
\hline \multirow{2}{*}{$\begin{array}{l}\text { Drunkenness before } \\
\text { military service }\end{array}$} & $<1$ time per week & $1075(26 ; 6)$ & 1 (Referent) & 1 (Referent) & 1 (Referent) & 1 (Referent) \\
\hline & $\geq 1$ time per week & $313(31 ; 9)$ & $1.3(1.0-1.6)$ & $1.1(0.8-1.4)$ & $1.6(1.0-2.5)$ & $1.1(0.7-1.9)$ \\
\hline \multirow{4}{*}{$\begin{array}{l}\text { Sweating exercise } \\
\text { (Brisk leisure time sport) }\end{array}$} & $\geq 3$ times per week & $438(27 ; 6)$ & 1 (Referent) & 1 (Referent) & 1 (Referent) & 1 (Referent) \\
\hline & 1-2 times per week & $415(28 ; 5)$ & $1.0(0.8-1.3)$ & $1.0(0.7-1.3)$ & $0.8(0.5-1.4)$ & $0.7(0.4-1.2)$ \\
\hline & Only leisured exercise & $257(27 ; 7)$ & $1.1(0.8-1.5)$ & $0.9(0.7-1.3)$ & $1.2(0.7-2.2)$ & $0.9(0.4-1.7)$ \\
\hline & No physical exercise & $278(27 ; 7)$ & $1.1(0.9-1.5)$ & $1.0(0.7-1.4)$ & $1.3(0.7-2.3)$ & $0.8(0.4-1.6)$ \\
\hline \multirow[t]{2}{*}{ Belongs to a sports club } & Yes, active member & $206(30 ; 6)$ & 1 (Referent) & 1 (Referent) & 1 (Referent) & 1 (Referent) \\
\hline & No & $1177(27 ; 6)$ & $0.9(0.7-1.2)$ & $0.9(0.7-1.2)$ & $1.1(0.6-2.0)$ & $1.1(0.6-2.1)$ \\
\hline \multirow[t]{2}{*}{ Participates in competitive sports } & Yes & $180(31 ; 6)$ & 1 (Referent) & 1 (Referent) & 1 (Referent) & 1 (Referent) \\
\hline & No & $1206(27 ; 8)$ & $0.9(0.7-1.2)$ & $0.8(0.6-1.1)$ & $0.8(0.5-1.5)$ & $0.8(0.4-1.4)$ \\
\hline \multirow[t]{2}{*}{ Last degree in school sports } & Good or excellent & $1101(28 ; 6)$ & 1 (Referent) & 1 (Referent) & 1 (Referent) & 1 (Referent) \\
\hline & Poor or fair & $286(24 ; 6)$ & $1.0(0.8-1.3)$ & $0.9(0.7-1.2)$ & $1.1(0.7-1.9)$ & $0.7(0.4-1.4)$ \\
\hline
\end{tabular}

Variable distribution was charted in 1411 male conscripts during the first week of military service and discharge outcomes were registered during the following

6-month military service.

Statistically significant $(p<0.05)$ findings are indicated with bold type.

${ }^{*}$ Adjusted for age (univariate).

**Acute: **Adjusted for age, company, smoking (previous or current smoker), frequency of drunkenness, baseline medical conditions (sum factor of earlier musculoskeletal symptoms, sports injury during the last month before entering the military), school success (educational level and grades combined), urbanisation level of the place of residence, waist circumference and physical fitness measured as a standing long jump test result. ${ }^{1}$ Attended upper secondary school, polytechnic, or university and reported excellent or good grades.

${ }^{2}$ Other subjects from upper secondary school, polytechnic, or university and conscripts from vocational school whose grades were excellent or good. ${ }^{3}$ Respondents with poorer grades in vocational school.

${ }^{4}$ Attended only comprehensive school or had permanently interrupted vocational or upper elementary school.

${ }^{5}$ Not adjusted by waist circumference since BMI and WC are strongly interconnected ( $\mathrm{2} 2$-test, $\mathrm{p}<0.001$ ).

${ }^{6}$ 'Minimal symptoms': maximum of 7 days in one anatomical region during the last month before military entry.

7'Mild symptoms': maximum of 7 days in 2 to 6 anatomical regions during the last month before military entry.

${ }^{8}$ 'Clear symptoms': included the remaining conscripts ie those with symptoms more than 8 days or more.

${ }^{9}$ Not adjusted by frequency of drunkenness since drunkenness and use of alcohol are strongly interconnected ( $x 2$-test, $\mathrm{p}<0.001$ ). 
Table 3 Hazard ratios (HR) for any overuse injury (OI) and severe overuse injury (severe OI) incidence by most important socioeconomic, health and health behaviour variables at baseline

\begin{tabular}{|c|c|c|c|c|c|c|}
\hline Baseline variable & Category & $\begin{array}{l}\text { Total number } \\
\text { (\% of experienced Ol; } \\
\% \text { of experienced } \\
\text { severe OI) }\end{array}$ & $\begin{array}{l}\text { HR for Ol incidence } \\
(n=721)^{*}\end{array}$ & $\begin{array}{l}\text { HR for Ol incidence } \\
(n=634)^{* *}\end{array}$ & $\begin{array}{l}\text { HR for severe OI } \\
\text { incidence }(\geq 7 \text { service } \\
\text { days lost })(n=251)^{*}\end{array}$ & $\begin{array}{l}\text { HR for sever } \\
\text { incidence }(\geq 7 \\
\text { days lost) ( }\end{array}$ \\
\hline \multicolumn{7}{|l|}{ Socioeconomic variables } \\
\hline \multirow[t]{2}{*}{ Age } & 18-19 years & $1052(50 ; 17)$ & 1 (Referent) & 1 (Referent) & 1 (Referent) & 1 (Referent) \\
\hline & 20-28 years & $359(54 ; 19)$ & $1.2(1.0-1.4)$ & $1.2(1.0-1.5)$ & $1.2(0.9-1.6)$ & $1.1(0.8-1.5)$ \\
\hline \multirow[t]{3}{*}{ Father's occupation } & Not physical & $488(49 ; 16)$ & 1 (Referent) & 1 (Referent) & 1 (Referent) & 1 (Referent) \\
\hline & Physical & $590(51 ; 19)$ & $1.1(0.9-1.3)$ & $1.0(0.8-1.2)$ & $1.2(0.9-1.6)$ & $1.1(0.8-1.5)$ \\
\hline & Unclear or unemployed & $302(55 ; 19)$ & $1.3(1.0-1.6)$ & $1.3(1.0-1.6)$ & $1.2(0.8-1.7)$ & $1.3(0.9-1.8)$ \\
\hline \multirow{4}{*}{$\begin{array}{l}\text { School success (educational level } \\
\text { and grades combined) }\end{array}$} & Excellent $^{1}$ & $218(36 ; 10)$ & 1 (Referent) & 1 (Referent) & 1 (Referent) & 1 (Referent) \\
\hline & $\operatorname{Good}^{2}$ & $608(53 ; 18)$ & $1.7(1.4-2.2)$ & $1.6(1.2-2.0)$ & $1.9(1.2-3.1)$ & $1.5(0.9-2.4)$ \\
\hline & Satisfactory $^{3}$ & $467(54 ; 21)$ & $1.9(1.5-2.4)$ & $1.4(1.1-1.9)$ & $2.4(1.5-3.9)$ & $1.4(0.9-2.4)$ \\
\hline & Poor $^{4}$ & $96(60 ; 22)$ & $2.4(1.7-3.4)$ & $1.9(1.3-2.7)$ & $2.8(1.5-5.1)$ & $1.9(1.0-3.6)$ \\
\hline \multirow[t]{4}{*}{ Company } & Anti-tank company & $263(52 ; 17)$ & 1 (Referent) & 1 (Referent) & 1 (Referent) & 1 (Referent) \\
\hline & Signal company & $540(56 ; 20)$ & $1.2(1.0-1.4)$ & $1.2(0.9-1.4)$ & $1.3(0.9-1.8)$ & $1.2(0.8-1.7)$ \\
\hline & Mortar company & $363(39 ; 12)$ & $0.9(0.7-1.1)$ & $1.0(0.7-1.2)$ & $0.9(0.6-1.4)$ & $0.8(0.5-1.3)$ \\
\hline & Engineer company & $245(56 ; 21)$ & $1.1(0.9-1.4)$ & $1.2(0.9-1.5)$ & $1.3(0.9-1.9)$ & $1.2(0.8-1.9)$ \\
\hline \multicolumn{7}{|l|}{ Health variables } \\
\hline \multirow[t]{4}{*}{ Body mass index $\left(\mathrm{BMl}=(\mathrm{kg}) /(\mathrm{m})^{2}\right)$} & Underweight $(<18.5)$ & $56(61 ; 25)$ & $1.5(1.1-2.1)$ & $1.7(1.1-2.4)$ & $1.8(1.0-3.1)$ & $2.0(1.1-3.5)$ \\
\hline & Normal $(18.5-25.0)$ & $812(49 ; 16)$ & 1 (Referent) & 1 (Referent) & 1 (Referent) & 1 (Referent) \\
\hline & Pre-obese $(25.0-30.0)$ & $300(49 ; 13)$ & $1.0(0.8-1.2)$ & $1.0(0.8-1.2)$ & $0.8(0.6-1.1)$ & $0.8(0.5-1.1)$ \\
\hline & Obese $(\geq 30.0)$ & $104(60 ; 25)$ & $1.4(1.1-1.9)$ & $1.3(1.0-1.8)$ & $1.7(1.1-2.5)$ & $1.3(0.8-2.2)$ \\
\hline \multirow[t]{4}{*}{ Waist circumference (WC, cm) } & Thin $(<80 \mathrm{~cm})$ & $271(48 ; 17)$ & $1.0(0.8-1.3)$ & $1.1(0.9-1.3)$ & $1.2(0.9-1.7)$ & $1.2(0.9-1.8)$ \\
\hline & Average $(80-93.5 \mathrm{~cm})$ & $739(50 ; 16)$ & 1 (Referent) & 1 (Referent) & 1 (Referent) & 1 (Referent) \\
\hline & Increased $(94-101.5 \mathrm{~cm})$ & $178(52 ; 15)$ & $1.1(0.9-1.4)$ & $1.1(0.8-1.4)$ & $0.9(0.6-1.4)$ & $0.8(0.5-1.3)$ \\
\hline & High ( $\geq 102 \mathrm{~cm}$ ) & $122(58 ; 24)$ & $1.4(1.1-1.8)$ & $1.5(1.1-2.0)$ & $1.7(1.1-2.6)$ & $1.7(1.1-2.7)$ \\
\hline \multirow[t]{2}{*}{ Self-assessed health ${ }^{6}$} & Good or very good & $743(47 ; 14)$ & 1 (Referent) & 1 (Referent) & 1 (Referent) & 1 (Referent) \\
\hline & Average or inferior & $646(56 ; 22)$ & $1.5(1.3-1.7)$ & $1.1(0.9-1.3)$ & $1.9(1.4-2.4)$ & $1.2(0.9-1.6)$ \\
\hline \multirow[t]{3}{*}{ Sum factor of musculoskeletal symptoms } & Minimal symptoms ${ }^{7}$ & $440(42 ; 10)$ & 1 (Referent) & 1 (Referent) & 1 (Referent) & 1 (Referent) \\
\hline & Mild symptoms ${ }^{8}$ & $548(51 ; 18)$ & $1.4(1.2-1.7)$ & $1.5(1.2-1.9)$ & $1.9(1.3-2.8)$ & $2.1(1.4-3.2)$ \\
\hline & Clear symptoms $^{9}$ & $400(61 ; 26)$ & $2.0(1.6-2.4)$ & $1.9(1.6-2.4)$ & $3.1(2.2-4.4)$ & $3.3(2.2-5.0)$ \\
\hline \multirow[t]{2}{*}{ Chronic disease } & No & $1012(50 ; 17)$ & 1 (Referent) & 1 (Referent) & 1 (Referent) & 1 (Referent) \\
\hline & Yes & $377(53 ; 20)$ & $1.1(0.9-1.3)$ & $1.1(0.9-1.3)$ & $1.2(0.9-1.6)$ & $1.2(0.9-1.6)$ \\
\hline
\end{tabular}


Table 3 Hazard ratios (HR) for any overuse injury (OI) and severe overuse injury (severe Ol) incidence by most important socioeconomic, health and health behaviour variables at baseline (Continued)

\begin{tabular}{|c|c|c|c|c|c|c|}
\hline \multirow[t]{2}{*}{ Orthopaedic surgery } & Never & $1273(50 ; 17)$ & 1 (Referent) & 1 (Referent) & 1 (Referent) & 1 (Referent) \\
\hline & Yes & $114(60 ; 24)$ & $1.3(1.0-1.6)$ & $1.3(1.0-1.7)$ & $1.4(1.0-2.1)$ & $1.5(1.0-2.3)$ \\
\hline \multirow[t]{2}{*}{ Chronic impairment or disability ${ }^{10}$} & No & $1165(49 ; 16)$ & 1 (Referent) & 1 (Referent) & 1 (Referent) & 1 (Referent) \\
\hline & Yes & $219(60 ; 26)$ & $1.5(1.2-1.8)$ & $1.2(1.0-1.5)$ & $1.8(1.4-2.4)$ & $1.5(1.1-2.1)$ \\
\hline \multirow[t]{2}{*}{ Sports injury during last month } & No & $1254(50 ; 17)$ & 1 (Referent) & 1 (Referent) & 1 (Referent) & 1 (Referent) \\
\hline & Yes & $130(65 ; 23)$ & $1.5(1.2-1.9)$ & $1.4(1.1-1.9)$ & $1.4(1.0-2.1)$ & $1.3(0.8-1.9)$ \\
\hline \multicolumn{7}{|l|}{ Health behaviour variables } \\
\hline \multirow[t]{2}{*}{ Smoking habits } & Never smoked regularly & $735(46 ; 15)$ & 1 (Referent) & 1 (Referent) & 1 (Referent) & 1 (Referent) \\
\hline & Has smoked regularly & $650(57 ; 21)$ & $1.4(1.2-1.6)$ & $1.1(0.9-1.3)$ & $1.6(1.2-2.0)$ & $1.2(0.9-1.6)$ \\
\hline \multirow[t]{2}{*}{ Use of alcohol ${ }^{11}$} & $<3$ times per week & $1148(49 ; 17)$ & 1 (Referent) & 1 (Referent) & 1 (Referent) & 1 (Referent) \\
\hline & $\geq 3$ times per week & $240(61 ; 23)$ & $1.3(1.1-1.6)$ & $1.2(1.0-1.4)$ & $1.4(1.0-1.9)$ & $1.2(0.8-1.6)$ \\
\hline \multirow[t]{2}{*}{ Drunkenness before military service } & $<1$ time per week & $1075(48 ; 17)$ & 1 (Referent) & 1 (Referent) & 1 (Referent) & 1 (Referent) \\
\hline & $\geq 1$ time per week & $313(60 ; 19)$ & $1.4(1.2-1.7)$ & $1.4(1.2-1.7)$ & $1.2(0.9-1.6)$ & $1.1(0.8-1.5)$ \\
\hline \multirow[t]{4}{*}{ Sweating exercise (Brisk leisure time sport) } & $\geq 3$ times per week & $438(42 ; 13)$ & 1 (Referent) & 1 (Referent) & 1 (Referent) & 1 (Referent) \\
\hline & 1-2 times per week & $415(54 ; 17)$ & $1.4(1.1-1.7)$ & $1.2(1.0-1.5)$ & $1.3(0.9-1.9)$ & $1.1(0.8-1.7)$ \\
\hline & Only leisured exercise & $257(55 ; 19)$ & $1.6(1.3-2.1)$ & $1.3(1.0-1.7)$ & $1.6(1.1-2.4)$ & $1.0(0.7-1.6)$ \\
\hline & No physical exercise & $278(57 ; 26)$ & $1.9(1.5-2.3)$ & $1.3(1.0-1.7)$ & $2.5(1.8-3.6)$ & $1.7(1.1-2.6)$ \\
\hline \multirow[t]{2}{*}{ Belongs to a sports club } & Yes, active member & $206(40 ; 14)$ & 1 (Referent) & 1 (Referent) & 1 (Referent) & 1 (Referent) \\
\hline & No & $1177(53 ; 18)$ & $1.6(1.3-2.0)$ & $1.5(1.2-2.0)$ & $1.5(1.0-2.2)$ & $1.2(0.8-1.8)$ \\
\hline \multirow[t]{2}{*}{ Participates in competitive sports } & No & $1206(51 ; 19)$ & 1 (Referent) & 1 (Referent) & 1 (Referent) & 1 (Referent) \\
\hline & Yes & $180(51 ; 17)$ & $0.9(0.7-1.1)$ & $1.5(1.1-2.1)$ & $1.0(0.7-1.5)$ & $1.9(1.2-3.0)$ \\
\hline \multirow[t]{2}{*}{ Last degree in school sports } & Good or excellent & $1101(49 ; 16)$ & 1 (Referent) & 1 (Referent) & 1 (Referent) & 1 (Referent) \\
\hline & Poor or fair & $286(59 ; 23)$ & $1.5(1.3-1.8)$ & $1.2(1.0-1.5)$ & $1.6(1.2-2.2)$ & $1.2(0.8-1.6)$ \\
\hline
\end{tabular}

Variable distribution was charted in 1411 male conscripts during the first week of military service and discharge outcomes were registered during the following 6-month military service. Statistically significant $(p<0.05)$ findings are indicated with bold type.

*Adjusted for age (univariate).

**Adjusted for age, company, smoking (previous or current smoker), frequency of drunkenness, baseline medical conditions (sum factor of earlier musculoskeletal symptoms and sports injury during the last month before military entry, previous orthopaedic operations), school success (educational level and grades combined), father's occupational group, belonging to a sport's club, waist circumference and physical fitness measured as a standing long jump test result. ${ }^{1}$ Attended upper secondary school, polytechnic, or university and reported excellent or good grades.

${ }^{2}$ Other subjects from upper secondary school, polytechnic, or university and conscripts from vocational school whose grades were excellent or good.

${ }^{3}$ Respondents with poorer grades in vocational school.

${ }^{4}$ Attended only comprehensive school or had interrupted vocational or upper elementary school.

${ }^{5}$ Attended only comprehensive school or had interrupted vocational or upper elementary school.
${ }^{5}$ bo waist circumference since BMI and WC are strongly interconnected ( $x 2$-test, $p<0.001$ ).

${ }^{5}$ Not adjusted by waist circun

${ }^{7}$ Minimal symptoms': maximum of 7 days in one anatomical region during the last month before military entry.

${ }^{8}$ 'Mild symptoms': maximum of 7 days in 2 to 6 anatomical regions during the last month before military entry.

${ }^{9}$ 'Clear symptoms': included the remaining conscripts i.e. those with symptoms more than 8 days or more.

${ }^{10}$ Due to earlier musculoskeletal injury.

${ }^{11}$ Not adjusted by frequency of drunkenness since drunkenness and use of alcohol are strongly interconnected ( $x 2$-test, $\left.\mathrm{p}<0.001\right)$. 
Table 4 Hazard ratios (HR) for any acute injury (AI) and severe acute injury (severe Al) incidence by physical fitness variables at baseline

\begin{tabular}{|c|c|c|c|c|c|c|}
\hline Baseline variable & Category & $\begin{array}{l}\text { Total number } \\
\text { (\% of experienced } \\
\mathrm{Al} \text { \% of experienced } \\
\text { severe } \mathrm{Al} \text { ) }\end{array}$ & $\begin{array}{l}\text { HR for Al incidence } \\
(\mathrm{n}=386)^{*}\end{array}$ & $\begin{array}{l}\text { HR for Al incidence } \\
(n=386)^{* *}\end{array}$ & $\begin{array}{l}\text { HR for severe Al } \\
\text { incidence }(\geq 7 \text { service } \\
\text { days lost) }(n=89)^{*}\end{array}$ & $\begin{array}{l}\text { HR for severe Al } \\
\text { incidence }(\geq 7 \text { service } \\
\text { days lost) }(n=89)^{* *}\end{array}$ \\
\hline \multirow[t]{2}{*}{ Self-assessed physical fitness $^{1}$} & Good or very good & $323(29 ; 6)$ & 1 (Referent) & 1 (Referent) & 1 (Referent) & 1 (Referent) \\
\hline & Average or inferior & $1066(27 ; 6)$ & $1.0(0.8-1.3)$ & $0.9(0.7-1.1)$ & $1.2(0.7-2.0)$ & $0.8(0.5-1.5)$ \\
\hline \multirow[t]{4}{*}{ Cooper's test (12-min running test) } & Excellent ( $\geq 3000 \mathrm{~m})$ & $51(22 ; 0)$ & 1 (Referent) & 1 (Referent) & 1 (Referent) & 1 (Referent) \\
\hline & Good ( $\geq 2600 \mathrm{~m})$ & $330(26 ; 5)$ & 1 (Referent) & 1 (Referent) & 1 (Referent) & 1 (Referent) \\
\hline & Fair good ( $\geq 2200 \mathrm{~m})$ & $630(28 ; 7)$ & $1.2(0.9-1.5)$ & $1.1(0.8-1.4)$ & $1.6(0.9-2.9)$ & $1.3(0.7-2.3)$ \\
\hline & Poor $(<2200 \mathrm{~m})$ & $358(28 ; 7)$ & $1.3(1.0-1.7)$ & $1.2(0.9-1.7)$ & $1.9(1.0-3.5)$ & $1.3(0.6-2.7)$ \\
\hline \multirow[t]{4}{*}{ Push-up test (repeats per 60 seconds) } & Excellent $(\geq 38)$ & $450(26 ; 5)$ & 1 (Referent) & 1 (Referent) & 1 (Referent) & 1 (Referent) \\
\hline & Good $(\geq 30)$ & $312(25 ; 4)$ & $0.9(0.7-1.3)$ & $0.9(0.7-1.2)$ & $0.9(0.4-1.7)$ & $0.7(0.4-1.5)$ \\
\hline & Fair good $(\geq 22)$ & $350(28 ; 7)$ & $1.1(0.8-1.5)$ & $1.0(0.7-1.3)$ & $1.4(0.8-2.5)$ & $0.9(0.5-1.7)$ \\
\hline & Poor $(<22)$ & $266(32 ; 10)$ & $1.4(1.1-1.9)$ & $1.2(0.9-1.6)$ & $2.2(1.3-3.9)$ & $1.7(0.9-3.1)$ \\
\hline \multirow{4}{*}{$\begin{array}{l}\text { Standing long jump test } \\
\text { (two attempts, best result) }\end{array}$} & Excellent $(\geq 2,40 \mathrm{~m})$ & $241(22 ; 3)$ & 1 (Referent) & 1 (Referent) & 1 (Referent) & 1 (Referent) \\
\hline & Good $(\geq 2,20 \mathrm{~m})$ & $363(28 ; 6)$ & $1.3(0.9-1.8)$ & $1.2(0.8-1.6)$ & $1.8(0.8-4.1)$ & $1.6(0.7-3.7)$ \\
\hline & Fair good ( $\geq 2,00 \mathrm{~m})$ & $442(30 ; 6)$ & $1.4(1.0-1.9)$ & $1.2(0.9-1.7)$ & $1.9(0.9-4.2)$ & $1.7(0.7-3.8)$ \\
\hline & Poor $(<2,00 \mathrm{~m})$ & $332(28 ; 9)$ & $1.4(1.0-2.0)$ & $1.2(0.9-1.8)$ & $3.3(1.5-7.1)$ & $2.8(1.2-6.4)$ \\
\hline \multirow[t]{4}{*}{ Conscript's muscle fitness index ${ }^{2}$} & Excellent (13-15 points) & $169(21 ; 4)$ & 1 (Referent) & 1 (Referent) & 1 (Referent) & 1 (Referent) \\
\hline & Good (9-12 points) & $361(30 ; 6)$ & $1.5(1.0-2.2)$ & $1.4(1.0-2.1)$ & $1.7(0.7-4.3)$ & $1.6(0.6-4.0)$ \\
\hline & Fair good (5-8 points) & $472(27 ; 6)$ & $1.4(0.9-2.0)$ & $1.2(0.8-1.8)$ & $1.9(0.8-4.6)$ & $1.4(0.6-3.6)$ \\
\hline & Poor (0-4 points) & $376(29 ; 8)$ & $1.6(1.1-2.4)$ & $1.3(0.9-2.0)$ & $2.6(1.1-6.3)$ & $1.8(0.7-4.6)$ \\
\hline \multirow[t]{4}{*}{ Conscript's physical fitness index ${ }^{3}$} & Excellent $(\geq 21,00)$ & $69(16 ; 0)$ & 1 (Referent) & 1 (Referent) & 1 (Referent) & 1 (Referent) \\
\hline & Good (17.00 - 20.99) & $409(29 ; 6)$ & 1 (Referent) & 1 (Referent) & 1 (Referent) & 1 (Referent) \\
\hline & Fair good (13.00 - 16.99) & $590(27 ; 7)$ & $1.0(0.8-1.3)$ & $0.9(0.7-1.1)$ & $1.5(0.9-2.4)$ & $1.1(0.6-1.9)$ \\
\hline & Poor $(<13.00)$ & $297(29 ; 8)$ & $1.2(0.9-1.6)$ & $1.0(0.7-1.4)$ & $1.9(1.1-3.4)$ & $1.3(0.6-2.6)$ \\
\hline \multirow{4}{*}{$\begin{array}{l}\text { Combination of standing long jump } \\
\text { and push-up test }\end{array}$} & Excellent $^{4}$ & $144(18 ; 2)$ & 1 (Referent) & 1 (Referent) & 1 (Referent) & 1 (Referent) \\
\hline & Good $^{5}$ & $447(30 ; 6)$ & $1.7(1.1-2.6)$ & $1.4(0.9-2.2)$ & $3.0(0.9-9.8)$ & $2.5(0.8-8.4)$ \\
\hline & Fair good ${ }^{6}$ & $650(27 ; 6)$ & $1.6(1.1-2.4)$ & $1.3(0.8-2.0)$ & $3.0(0.9-9.6)$ & $2.1(0.6-6.9)$ \\
\hline & Poor ${ }^{7}$ & $137(33 ; 14)$ & $2.5(1.5-4.0)$ & $1.8(1.0-3.0)$ & $8.8(2.6-29.8)$ & $5.9(1.6-21.3)$ \\
\hline
\end{tabular}


Table 4 Hazard ratios (HR) for any acute injury (AI) and severe acute injury (severe Al) incidence by physical fitness variables at baseline (Continued)

\begin{tabular}{|c|c|c|c|c|c|c|}
\hline \multirow{4}{*}{$\begin{array}{l}\text { Combination of standing long jump } \\
\text { and Cooper's test }\end{array}$} & Excellent $^{4}$ & $136(21 ; 2)$ & 1 (Referent) & 1 (Referent) & 1 (Referent) & 1 (Referent) \\
\hline & Good $^{5}$ & $504(28 ; 6)$ & $1.4(0.9-2.1)$ & $1.4(0.9-2.0)$ & $3.0(0.9-9.8)$ & $2.7(0.8-9.0)$ \\
\hline & Fair good ${ }^{6}$ & $550(27 ; 6)$ & $1.4(0.9-2.1)$ & $1.2(0.8-1.9)$ & $2.8(0.9-9.2)$ & $2.4(0.7-8.1)$ \\
\hline & Poor $^{7}$ & $175(31 ; 12)$ & $1.9(1.2-2.9)$ & $1.7(1.0-2.8)$ & $7.0(2.1-23.6)$ & $5.8(1.6-21.3)$ \\
\hline
\end{tabular}

Variable distribution was charted in 1411 male conscripts during the first two weeks of military service and discharge outcomes were registered during the following 6-month military service.

Statistically significant $(p<0.05)$ findings are indicated with bold type.

*Adjusted for age (univariate).

${ }^{*}$ Adjusted for age, company, smoking (previous or current smoker), frequency of drunkenness, baseline medical conditions (sum factor of earlier musculoskeletal symptoms, sports injury during the last month before entering the military), school success (educational level and grades combined), urbanisation level of the place of residence and waist circumference.

${ }^{1}$ Compared to age mates.

${ }^{2}$ Muscle fitness index is the sum of individual muscle fitness test results including push-up, sit-up, pull-up, standing long jump and back lift tests.

${ }^{3}$ Conscript's physical fitness index $(\mathrm{CPFI})=(12$-min running test result $(\mathrm{m})+100 \times$ muscle fitness test points) $/ 200$.

${ }^{4}$ Excellent or good result in Cooper's test and excellent result in selected muscular fitness test.
${ }^{5}$ Excellent result in selected muscular fitness test and fair good or poor result in Cooper's test; or excellent result in Cooper's test and good, fair good, or poor result in selected muscular fitness test.

'Excellent result in selected muscular fitness test and fair good or poor result in Cooper's test; or excellent result in Cooper's test and good, fair good, or poor result in selected mil in
or good result in Cooper's test and good or fair good result in selected muscular fitness test; or fair good result in Cooper's test and good result in selected muscular fitness test.

or good result in Cooper's test and good or fair good result in selected muscular fitness test;
${ }^{6}$ Poorer results than aforementioned, except the combination of poor results in both tests.

${ }^{7}$ Poor result in both tests. 
Table 5 Hazard ratios (HR) for any overuse injury (OI) and severe overuse injury (severe OI) incidence by physical fitness variables at baseline

\begin{tabular}{|c|c|c|c|c|c|c|}
\hline Baseline variable & Category & $\begin{array}{l}\text { Total number } \\
\text { (\% of experienced OI; } \\
\% \text { of experienced } \\
\text { severe OI) }\end{array}$ & $\begin{array}{l}\text { HR for Ol incidence } \\
(\mathrm{n}=721)^{*}\end{array}$ & $\begin{array}{l}\text { HR for Ol incidence } \\
(\mathrm{n}=721)^{* *}\end{array}$ & $\begin{array}{l}\text { HR for severe OI } \\
\text { incidence } \\
(\geq 7 \text { service days lost }) \\
(\mathrm{n}=251)^{*}\end{array}$ & $\begin{array}{l}\text { HR for severe OI } \\
\text { incidence } \\
(\geq 7 \text { service days lost }) \\
(\mathrm{n}=251)^{* *}\end{array}$ \\
\hline \multirow[t]{2}{*}{ Self-assessed physical fitness $^{1}$} & Good or very good & $323(46 ; 14)$ & 1 (Referent) & 1 (Referent) & 1 (Referent) & 1 (Referent) \\
\hline & Average or inferior & $1066(53 ; 19)$ & $1.4(1.1-1.6)$ & $1.1(0.9-1.3)$ & $1.5(1.1-2.1)$ & $1.0(0.7-1.5)$ \\
\hline \multirow[t]{4}{*}{ Cooper's test (12-min running test) } & Excellent ( $\geq 3000 \mathrm{~m})$ & $51(43 ; 12)$ & 1 (Referent) & 1 (Referent) & 1 (Referent) & 1 (Referent) \\
\hline & Good ( $\geq 2600 \mathrm{~m})$ & $330(44 ; 13)$ & 1 (Referent) & 1 (Referent) & 1 (Referent) & 1 (Referent) \\
\hline & Fair good ( $\geq 2200 \mathrm{~m})$ & $630(50 ; 15)$ & $1.3(1.1-1.5)$ & $1.1(0.9-1.3)$ & $1.2(0.9-1.7)$ & $1.1(0.7-1.6)$ \\
\hline & Poor (<2200 m) & $358(59 ; 26)$ & $1.8(1.5-2.2)$ & $1.4(1.1-1.8)$ & $2.4(1.7-3.4)$ & $1.9(1.3-3.0)$ \\
\hline \multirow[t]{4}{*}{ Push-up test (repeats per 60 seconds) } & Excellent ( $\geq 38)$ & $450(48 ; 14)$ & 1 (Referent) & 1 (Referent) & 1 (Referent) & 1 (Referent) \\
\hline & Good $(\geq 30)$ & $312(52 ; 19)$ & $1.2(1.0-1.5)$ & $1.1(0.8-1.3)$ & $1.3(0.9-1.9)$ & $1.1(0.7-1.6)$ \\
\hline & Fair good $(\geq 22)$ & $350(49 ; 17)$ & $1.1(0.9-1.4)$ & $1.0(0.8-1.2)$ & $1.3(0.9-1.8)$ & $0.9(0.6-1.4)$ \\
\hline & Poor $(<22)$ & $266(57 ; 22)$ & $1.5(1.2-1.8)$ & $1.1(0.8-1.4)$ & $1.7(1.2-2.5)$ & $1.0(0.7-1.5)$ \\
\hline \multirow{4}{*}{$\begin{array}{l}\text { Standing long jump test (two attempts, } \\
\text { best result) }\end{array}$} & Excellent ( $\geq 2,40 \mathrm{~m})$ & $241(45 ; 12)$ & 1 (Referent) & 1 (Referent) & 1 (Referent) & 1 (Referent) \\
\hline & $\operatorname{Good}(\geq 2,20 \mathrm{~m})$ & $363(50 ; 17)$ & $1.2(0.9-1.5)$ & $1.1(0.9-1.4)$ & $1.6(1.0-2.5)$ & $1.5(0.9-2.4)$ \\
\hline & Fair good ( $\geq 2,00 \mathrm{~m})$ & $442(52 ; 17)$ & $1.2(0.9-1.6)$ & $1.1(0.9-1.4)$ & $1.5(1.0-2.3)$ & $1.4(0.9-2.2)$ \\
\hline & Poor $(<2,00 \mathrm{~m})$ & $332(56 ; 23)$ & $1.6(1.2-2.0)$ & $1.2(0.9-1.6)$ & $2.3(1.5-3.6)$ & $1.8(1.1-3.0)$ \\
\hline \multirow[t]{4}{*}{ Back lift test (repeats per 60 seconds) } & Excellent $(\geq 60)$ & $660(46 ; 13)$ & 1 (Referent) & 1 (Referent) & 1 (Referent) & 1 (Referent) \\
\hline & Good $(\geq 50)$ & $284(50 ; 22)$ & $1.1(0.9-1.4)$ & $0.9(0.8-1.2)$ & $1.7(1.3-2.4)$ & $1.4(1.0-2.0)$ \\
\hline & Fair good $(\geq 40)$ & $291(54 ; 20)$ & $1.3(1.1-1.5)$ & $1.1(0.9-1.3)$ & $1.5(1.1-2.2)$ & $1.2(0.8-1.7)$ \\
\hline & Poor $(<40)$ & $143(68 ; 25)$ & $1.9(1.5-2.4)$ & $1.5(1.1-1.9)$ & $2.1(1.5-3.2)$ & $1.4(0.9-2.2)$ \\
\hline \multirow[t]{4}{*}{ Conscript's muscle fitness index ${ }^{2}$} & Excellent (13-15 points) & $169(43 ; 13)$ & 1 (Referent) & 1 (Referent) & 1 (Referent) & 1 (Referent) \\
\hline & Good (9-12 points) & $361(45 ; 13)$ & $1.2(0.9-1.6)$ & $1.1(0.9-1.5)$ & $1.0(0.6-1.7)$ & $0.9(0.6-1.6)$ \\
\hline & Fair good (5-8 points) & $472(53 ; 18)$ & $1.5(1.1-1.9)$ & $1.2(0.9-1.6)$ & $1.5(1.0-2.5)$ & $1.2(0.7-2.0)$ \\
\hline & Poor (0-4 points) & $376(57 ; 23)$ & $1.7(1.3-2.3)$ & $1.3(1.0-1.8)$ & $2.1(1.3-3.3)$ & $1.3(0.8-2.2)$ \\
\hline \multirow[t]{4}{*}{ Conscript's physical fitness index ${ }^{3}$} & Excellent $(\geq 21,00)$ & $69(39 ; 9)$ & 1 (Referent) & 1 (Referent) & 1 (Referent) & 1 (Referent) \\
\hline & Good (17.00 - 20.99) & $409(44 ; 13)$ & 1 (Referent) & 1 (Referent) & 1 (Referent) & 1 (Referent) \\
\hline & Fair good (13.00 - 16.99) & $590(53 ; 17)$ & $1.3(1.1-1.5)$ & $1.2(1.0-1.5)$ & $1.2(0.9-1.7)$ & $1.2(0.8-1.7)$ \\
\hline & Poor $(<13.00)$ & $297(59 ; 26)$ & $1.8(1.5-2.2)$ & $1.4(1.1-1.8)$ & $2.4(1.7-3.4)$ & $1.7(1.1-2.7)$ \\
\hline \multirow{4}{*}{$\begin{array}{l}\text { Combination of standing long jump } \\
\text { and back lift test }\end{array}$} & Excellent $^{4}$ & $177(45 ; 12)$ & 1 (Referent) & 1 (Referent) & 1 (Referent) & 1 (Referent) \\
\hline & $\operatorname{Good}^{5}$ & $523(46 ; 14)$ & $1.1(0.8-1.4)$ & $1.1(0.9-1.4)$ & $1.2(0.7-2.0)$ & $1.1(0.7-1.9)$ \\
\hline & Fair good ${ }^{6}$ & $607(54 ; 21)$ & $1.4(1.1-1.8)$ & $1.2(1.0-1.6)$ & $1.9(1.2-3.0)$ & $1.5(0.9-2.5)$ \\
\hline & Poor $^{7}$ & $71(70 ; 27)$ & $2.5(1.7-3.5)$ & $1.7(1.2-2.6)$ & $2.9(1.6-5.4)$ & $1.7(0.8-3.6)$ \\
\hline
\end{tabular}


Table 5 Hazard ratios (HR) for any overuse injury (OI) and severe overuse injury (severe OI) incidence by physical fitness variables at baseline (Continued)

\begin{tabular}{|c|c|c|c|c|c|c|}
\hline \multirow[t]{4}{*}{ Combination of back lift and push-up test } & Excellen $^{4}$ & $335(44 ; 13)$ & 1 (Referent) & 1 (Referent) & 1 (Referent) & 1 (Referent) \\
\hline & Good $^{5}$ & $440(48 ; 17)$ & $1.2(1.0-1.5)$ & $1.0(0.8-1.3)$ & $1.4(0.9-2.0)$ & $1.1(0.8-1.7)$ \\
\hline & Fair good ${ }^{6}$ & $536(55 ; 20)$ & $1.5(1.2-1.8)$ & $1.1(0.9-1.4)$ & $1.7(1.2-2.4)$ & $1.1(0.7-1.7)$ \\
\hline & Poor $^{7}$ & $67(67 ; 31)$ & $2.4(1.7-3.4)$ & $1.7(1.2-2.5)$ & $3.2(1.9-5.5)$ & $1.7(0.9-3.2)$ \\
\hline \multirow{4}{*}{$\begin{array}{l}\text { Combination of standing long jump } \\
\text { and Cooper's test }\end{array}$} & Excellent $^{4}$ & $136(42 ; 8)$ & 1 (Referent) & 1 (Referent) & 1 (Referent) & 1 (Referent) \\
\hline & Good $^{5}$ & $504(47 ; 15)$ & $1.3(1.0-1.7)$ & $1.1(0.8-1.5)$ & $2.1(1.1-4.0)$ & $1.9(1.0-3.6)$ \\
\hline & Fair good ${ }^{6}$ & $550(54 ; 19)$ & $1.6(1.2-2.1)$ & $1.3(0.9-1.7)$ & $2.6(1.4-4.8)$ & $2.0(1.1-3.9)$ \\
\hline & Poor ${ }^{7}$ & $175(59 ; 26)$ & $2.1(1.5-2.9)$ & $1.5(1.0-2.2)$ & $4.4(2.2-8.4)$ & $3.3(1.6-7.0)$ \\
\hline
\end{tabular}

Variable distribution was charted in 1411 male conscripts during the first two weeks of military service and discharge outcomes were registered during the following 6-month military service.

Statistically significant $(p<0.05)$ findings are indicated with bold type.

*Adjusted for age (univariate).

**Adjusted for age, company, smoking (previous or current smoker), frequency of drunkenness, baseline medical conditions (sum factor of earlier musculoskeletal symptoms and sports injury during the last month before military entry, previous orthopaedic operations), school success (educational level and grades combined), father's occupational group, belonging to a sport's club, waist circumference and physical fitness

measured as a standing long jump test result.

Compared to age mates.

${ }^{2}$ Muscle fitness index is the sum of individual muscle fitness test results including push-up, sit-up, pull-up, standing long jump and back lift tests.

${ }^{3}$ Conscript's physical fitness index $(\mathrm{CPFI})=(12$-min running test result $(\mathrm{m})+100 \times$ muscle fitness test points) $/ 200$.

${ }^{4}$ Excellent or good result in Cooper's test and excellent result in selected muscular fitness test

${ }^{5}$ Excellent result in selected muscular fitness test and fair good or poor result in Cooper's test; or excellent result in Cooper's test and good, fair good, or poor result in selected muscular fitness test;

or good result in Cooper's test and good or fair good result in selected muscular fitness test; or fair good result in Cooper's test and good result in selected muscular fitness test.

作

${ }^{7}$ Poor result in both tests. 
health behaviour (Table 3) and physical fitness variables (Table 5) in the age-adjusted and multivariate models.

Among socioeconomic background variables, poor school success, older age and unemployed or unclear father's occupational status were associated with $\mathrm{OI}$ incidence but not with severe OI (Table 3).

Considering health, pre-service musculoskeletal symptoms were clearly associated with incidence of OI and severe OI ( $\mathrm{HR}=3.3$; 95\% CI: $2.2-5.0)$ in a graded manner even after further adjustments. High waist circumference $(\geq 102 \mathrm{~cm})$ and, on the other hand, being underweight $(\mathrm{BMI}<18.5 \mathrm{~kg} / \mathrm{m} 2)$ increased the $\mathrm{HR}$ for both OI and severe OI (Table 3).

With regard to health behaviours, low pre-service PA remained predictive for incidence of OI and severe OI $(\mathrm{HR}=1.7 ; 95 \% \mathrm{CI}: 1.1-2.6)$ even after further adjustments including physical fitness in multivariate models. After multivariate adjustments drunkenness at least once per week before military entry was associated with OI incidence $(\mathrm{HR}=1.4 ; 95 \% \mathrm{CI}: 1.2-1.7)$. On the other hand, participation in competitive sports was also associated with OI and severe OI in multivariate models (Table 3).

Considering physical fitness single test items of all physical fitness tests were predictive for OI or severe OI. Associations between poor standing long-jump and poor 12-min running test results and severe OI remained predictive after multivariable adjustments. Similarly, poor results in 12-min running and back-lift test were associated with OI incidence (Table 5). Conscripts with poor level of fitness both in standing long-jump and 12-min running test had the highest $\mathrm{HR}$ for severe $\mathrm{OI}(\mathrm{HR}=3.3$; 95\% CI: 1.6-7.0) (Table 5).

\section{Discussion}

Of the 1411 participants, $27 \%$ sustained an acute injury (AI) and 51\% suffered from overuse injury (OI) during the 6-month service. Most injuries were in the lower extremities (67\%) followed by the back (18\%). Low levels of physical fitness and poor school success were associated with acute and overuse musculoskeletal injuries during military training in a graded manner. Low pre-service PA was associated with OIs but not with AIs. Interestingly, high waist circumference as a mark of abdominal obesity and low BMI, at the other extreme end, were associated with OIs indicating U-shaped relationship between OIs and body weight. Participation in competitive sports was a risk factor for OIs during military training, indicating that as the total amount of exercise increases from 17 hours per week in military setting, there is point at which OIs clearly increase.

In earlier military studies, low muscular endurance $[21,22,36]$ and especially low aerobic endurance [14,17-22] have been shown to be predictors of injuries. In the present study, standing long-jump test performed according to the standard protocol in the Finnish Defence Forces, proved to be a good indicator for both acute and overuse injuries. The test measures explosive force production of the lower limbs and motor control ability (Figure 3) [17]. Coimpairments in cardiorespiratory and muscular fitness may be an indicator of poor ability to perform longlasting weight bearing activities requiring both strength and aerobic capacity, such as loaded marching. Conscripts with lower cardiorespiratory fitness levels may perceive military training as more difficult and fatigue more rapidly [37]. Fatigue leads to changes in gait and kinematics in lower extremities [38,39]. This may induce musculoskeletal stress in specific body areas and predispose to injuries $[39,40]$. Hence, it is logical that poor results in 12-min running test predicted particularly OIs in the present study. Muscular fatigue may lead to a greater reliance on other muscle groups as the active muscle groups begin to fatigue [41]. In addition, adolescents with motor impairment have a reduced movement economy during aerobic exercise [42]. Probably, core stability as a subset of motor control [43] also has an important role in the muscular fatigue and pathogenesis of lower extremity injuries [44].

The present finding that low PA level before military entry is associated particularly with OIs is concordant with previous studies $[15,16,45,46]$ and suggests that untrained conscripts overload their musculoskeletal structures and tissues more often than their active counterparts during military training. Physical activity may result in adaptation of the body and thereby help to prevent overuse related injuries when the conscript is subjected to new strains $[18,47]$. The present findings indicate that basic military training exerts a load particularly on lower limbs and low back due to high amount of weight bearing activities with backpack.

Interestingly, variables which were most strongly associated with the outcomes of the present study, namely earlier school success and physical fitness, are largely interconnected and related to PA in previous studies among the young. According to a Swedish study [48], continuation of engaging in sports exercise after childhood was clearly associated with good school success and high volume of physical exercise in childhood. In contrast to education level, poor entry-level fitness of conscripts is a modifiable predictor of injuries and amenable to prevention programmes. In our previous studies, a neuromuscular exercise and injury prevention counseling programme was effective in preventing acute ankle and upper extremity injuries [49] as well as disability due to low back pain [50] in young male army conscripts. Because even good or excellent degree in earlier school sports was not a protective factor against AIs, preventive neuromuscular interventions are needed in the whole population. Our previous 
studies showed that this type of intervention programme is not effective in reducing OIs [51]. Ideally, prevention programmes that would have an effect on OIs should start well before entry to military and target to increase PA while decreasing passive sitting and screen time at the same time.

It is not easy to find ways to promote PA among the young. One of the most promising ways to increase PA and decrease sedentary behaviour is cycling to school instead of passive commuting [52]. Moreover, active commuting is associated with higher levels of PA in over 27 years of follow-up, and thus, may contribute to a healthy and active lifestyle through life-course [53]. Other method proven to increase PA among young is enhancing PA during school days by encouraging to move during breaks between lessons and while commuting school travels and by adding elements to lessons that increase PA (e.g. lessons outside, more group work) [54]. It has been clearly demonstrated in various surveys that less than half [55] or recently in a objectively measured study [56] that less than $10 \%$ of elementary school children meet the PA guidelines in developed countries. Moreover, sedentary time increases and PA decreases from lower elementary school to upper elementary school [56,57]. Probably by increasing the number of school sports lessons especially in upper elementary and high school or vocational schools would have positive effect on PA. In a society level, building pedestrian walkways and cycle paths and adding PA counselling have positive results in PA at a reasonable price [58-60]. Moreover, safety issues should not be neglected with these types of programmes.

In the present study, both the high BMI and high waist circumference as a marker of obesity were associated with $\mathrm{AI}$ and $\mathrm{OI}$ in age-adjusted models. However, the associations weakened in multivariate models, but high waist circumference remained its significance for OIs. The U-shaped association by Jones et al. [61], indicating that being underweight is a risk factor also, was partly observed. Underweight conscripts according to BMI had higher HR for OIs than conscripts with normal BMI. This association was not observed among conscripts with low waist circumference. In young men low BMI may also be an indicator of low lean body mass i.e. musculature explaining the finding. The limits for underweight/thin were stricter considering BMI categories than waist circumference categories, which also probably explains the different outcomes. Furthermore, the results indicate an association between low body weight and OIs only among the most lightest $(<3 \%$ of age cohort according to BMI, Table 3) group of young men. This is in consonance with previous studies reporting association between underweight and musculoskeletal injuries especially considering lower limb OIs and stress fractures during intensive military training $[20,61,62]$. The explanation may be that recruits with a higher BMI are able to cope better with load carriage tasks than their light counterparts $[63,64]$, because proportional weight increase is higher among light persons.

Obesity is clearly associated with decrease in physical fitness and increased risk for musculoskeletal injuries $[22,61,65]$ and healthcare usage [66] which leads to problems to meet military service standards $[22,67]$. Obesity impairs functional ability in everyday living and is associated with difficulties in physical demands with strenuous work and pain after the strain $[68,69]$. Obese people are impeded in sport activities, walking outdoors and up and down stairs, and in squatting, stooping and lifting [70]. High BMI alters body geometry and postural stability [71,72]. In turn, these alterations may reduce movement efficiency and increase the risk of injury [73]. Reducing weight improves the balance control in obese civilian men and decreases the risk of falling injuries [74].

Finnish compulsory military service reaching a vast majority of 19-year-old young men offers a unique opportunity for intervention against physical inactivity and obesity. Furthermore, in obese Finnish conscripts, military training assists in reducing body mass and improving cardiorespiratory fitness [75,76]. Moreover, conscripts may adopt healthy changes in nutrition and other lifestyle habits especially at the beginning of the military service [76].

The strengths of the study include the use of computerised patient files guaranteeing a consistent method for data acquisition because all patients who entered the garrison clinic were recorded. Second, the definitions of outcomes were clear and defined by ICD-10 codes set by an independent physician in the garrison clinic. Moreover, the patient files were manually checked to distinguish AIs and OIs. Third, the participation rate was high (98\%). Fourth, the military environment provided highly standardised conditions for investigating the effect of individual risk factors: Conscripts underwent daily military programmes that were nearly equal considering the time, intensity and quality of physical training providing equal opportunity for food supply, rest and sleep. Fifth, the design of the study was prospective. Finally, due to compulsory nature of military service in Finland, reaching annually about $80 \%$ of the age cohort entering into the service, the population-based sample of incoming conscripts formed a comprehensive sample of Finnish young male adults who had passed their medical examination performed by a physician before military entry.

Our study has also limitations. First, although the compulsory military service concerns all Finnish male citizens, approximately $7 \%$ of all eligible men choose to perform non-military service in Finland and approximately 15\% of conscripts are exempted from duty after physician examinations at the call-up or during the first week of military service due to minimum physical and mental requirements established for military service $[77,78]$. Second, the findings 
can only be generalised to young men because less than $3 \%$ of the conscripts were females and they were excluded from the analyses. Third, after the initial 8 weeks of basic training, the training programmes became more divergent due to the more specialised military tasks in each company. This also caused drop-out due to a company change $(\mathrm{n}=338)$ and significant proportion $(33.5 \%)$ of conscripts were not followed until the end of 180 days follow-up. On the other hand, all conscripts were followed up for the first 8 weeks of service and the results were adjusted by company. Finally, because the threshold for seeking medical care may vary between individuals, some conscripts may have been more inclined to seek professional care than others.

For the prevention of acute lower limb injuries, several practical neuromuscular training strategies are shown to be effective in team sports $[32,51,79,80]$. In military environment, pre-conditioning of low-fit recruits resulted in lower number of discharges and a tendency towards lower injury risk [81]. Similarly, modification of training programmes by reducing cumulative marching and by assuring enough recovery time decreased clearly OIs in Israeli and U.S. Armies $[82,83]$. More recently, Coppack and colleagues [84] completed a RCT-study of 1502 male and female recruits in UK. They reported that a 14-week training programme consisting of 4 warm-up exercises and 4 warm-down static stretches completed 7 times per week (total $105 \mathrm{mi}$ nutes per week) was effective in reducing overuse anterior knee pain (adjusted $\mathrm{HR}=0.25$; 95\% CI: 0.13-0.49). Another RCT-study conducted among Danish conscripts, revealed that concurrent exercise programme enhancing muscular strength, coordination, and flexibility was not effective in reducing the incidence of lower extremity OI. The intervention was speculated to be more effective in situations with a more gradual increase in load [85]. Dramatically increased number of low-fit and obese incoming conscripts during last decades forces the military training programmes to adapt to these new challenges $[28,67,78]$. Therefore, it has been suggested that the time frame for physical adjustment and development should be the whole duration of service. More progressive individual training programmes, coaching and goals could alleviate the problem of low-fit incoming conscripts [86].

Well-planned randomised controlled studies are needed to provide more evidence from effective interventions especially on the prevention of OIs. For example, studies investigating the effect of physical training programme in good time before entry into the compulsory military service are needed. The effect of the intervention programmes should be tested among those who are at the highest risk for musculoskeletal injuries. Moreover, more research is needed considering how positive results from evidence-based practice could be implemented into the injury prevention of everyday life.

\section{Conclusions}

In Finland, $80 \%$ of 19 -year-old men enter into the compulsory military service. Half of them suffer from OI and nearly one third sustain an AI during the 6-month military training. This study showed that a low cardiorespiratory and muscular fitness especially considering lower limb force production and motor control are associated with injuries. In addition, low pre-service PA and high waist circumference as a mark of abdominal obesity and, on the other hand, low BMI were associated with OIs indicating U-shaped relationship between OIs and body weight.

Poor entry-level cardiorespiratory and muscular fitness is a modifiable risk factor of injuries and amenable to prevention programmes. Because even good or excellent degree in earlier school sports was not a protective factor for AIs in military setting, preventive interventions are needed in all age groups. Neuromuscular training integrated to warm-up or cool-down sessions including balance and coordination exercises that enhance proprioceptive sensation may reduce the burden of acute PA related injuries in sports, in military training, in leisure time activities, as well as in school sport lessons. We suggest that in order to prevent OIs during intensive physical training more gradual onset of the training is needed among previously inactive and low-fit conscripts. A desirable goal in a pre-training programme before entering the military service could be a running distance of $2600 \mathrm{~m}$ or more in the 12-min running test. To distinguish young men with elevated risk on injuries before military entry, we suggest screening of all 9th grade students for low cardiorespiratory and muscular fitness.

\section{Competing interests}

The authors declare that they have no competing interests.

\section{Authors' contributions}

HT wrote the first draft of the manuscript. HT, JHS, PK and JP participated in data analysis, interpretation and data acquisition. JHS was the primary investigator together with JP. They initiated and conceptually designed the study and took part in manuscript reviewing. PK also participated in the study as a significant manuscript reviewer. HP participated in study concept and design as well as manuscript reviewing. JPR and JV took part in data analysis and interpretation and revised the manuscript critically. All authors have made substantive intellectual contributions to the study. All authors reviewed the article and gave the final approval of the manuscript.

\section{Acknowledgements}

This study was supported by the Centre for Military Medicine, Helsinki, Finland; the Scientific Advisory Board for Defence, Helsinki, Finland; the Ministry of Education; and the Medical Research Fund of the Tampere University Hospital, Tampere, Finland. We appreciate the excellent cooperation of the personnel of the Pori Brigade over the course of the study. We thank MSc. Kari Tokola for statistical advice, Anni Taanila for help in data acquisition, Petteri Vuorinen for the excellent collaboration between Pori Brigade and Tampere Research Center of Sports Medicine and Olli Ohrankämmen for the vital support in combining the data archives to interface with each other. We also thank director of the Centre for Military Medicine Jouko Peltomaa and research director Paula Kinnunen, for their support and encouragement. 


\section{Author details}

${ }^{1}$ Research Department, Centre for Military Medicine, Lahti and Helsinki, Finland. ${ }^{2}$ Tampere Research Centre of Sports Medicine, and Injury and Osteoporosis Research Center, UKK Institute, PO Box 30FIN-33501 Tampere, Finland. ${ }^{3}$ Research Unit of Pirkanmaa Hospital District, and Department of Orthopedics and Trauma Surgery, Tampere University Hospital, Tampere, Finland. ${ }^{4}$ Division of Orthopedics and Traumatology, Seinäjoki Central Hospital, Seinäjoki, Finland. ${ }^{5}$ University of Tampere, Seinäjoki, Finland. ${ }^{6}$ Training Division of the Defence Staff, Finnish Defense Forces, Helsinki, Finland.

Received: 1 October 2014 Accepted: 17 April 2015

\section{Published online: 01 May 2015}

\section{References}

1. Haskell WL, Blair SN, Hill JO. Physical activity: health outcomes and importance for public health policy. Prev Med. 2009;49(4):280-2

2. Physical activity guidelines advisory committee report. In. Washington (DC): U.S. Department of Health and Human Service.; 2008: http://www.health.gov/ paguidelines/report/A_Summary.aspx\#_Toc199951124. Accessed 1 May 2015.

3. Matthews CE, Chen KY, Freedson PS, Buchowski MS, Beech BM, Pate RR, et al. Amount of time spent in sedentary behaviors in the United States, 2003-2004. Am J Epidemiol. 2008;167(7):875-81.

4. Dunstan DW, Barr EL, Healy GN, Salmon J, Shaw JE, Balkau B, et al. Television viewing time and mortality: the Australian Diabetes, Obesity and Lifestyle Study (AusDiab). Circulation. 2010;121(3):384-91.

5. Katzmarzyk PT, Church TS, Craig CL, Bouchard C. Sitting time and mortality from all causes, cardiovascular disease, and cancer. Med Sci Sports Exerc. 2009;41(5):998-1005

6. Hurtig-Wennlof A, Ruiz JR, Harro M, Sjostrom M. Cardiorespiratory fitness relates more strongly than physical activity to cardiovascular disease risk factors in healthy children and adolescents: the European Youth Heart Study. Eur J Cardiovasc Prev Rehabil. 2007;14(4):575-81.

7. Blair SN, Cheng Y, Holder JS. Is physical activity or physical fitness more important in defining health benefits? Med Sci Sports Exerc. 2001;33(6 Suppl):S379-99. discussion S419-320.

8. Williams PT. Physical fitness and activity as separate heart disease risk factors: a meta-analysis. Med Sci Sports Exerc. 2001;33(5):754-61.

9. Macera CA, Jackson KL, Hagenmaier GW, Kronenfeld JJ, Kohl HW, Blair SN. Age, physical activity, physical fitness, body composition, and incidence of orthopedic problems. Res Q Exerc Sport. 1989;60(3):225-33.

10. Kaufman KR, Brodine S, Shaffer R. Military training-related injuries: surveillance, research, and prevention. Am J Prev Med. 2000;18(3 Suppl):54-63.

11. Pakzad-Vaezi K, Singhal A. Trends in paediatric sport- and recreation-related injuries: An injury surveillance study at the British Columbia Children's Hospital (Vancouver, British Columbia) from 1992 to 2005. Paediatr Child Health. 2011;16(4):217-21.

12. Parkkari J, Kannus $P$, Natri A, Lapinleimu I, Palvanen $M$, Heiskanen $M$, et al. Active living and injury risk. Int J Sports Med. 2004;25(3):209-16.

13. Tirikainen $\mathrm{K}$, Lounamaa A, Paavola M, Kumpula H, Parkkari J. Trend in sports injuries among young people in Finland. Int J Sports Med. 2008;29(6):529-36.

14. Knapik JJ, Swedler DI, Grier TL, Hauret KG, Bullock SH, Williams KW, et al. Injury reduction effectiveness of selecting running shoes based on plantar shape. J Strength Cond Res. 2009;23(3):685-97.

15. Shaffer RA, Brodine SK, Almeida SA, Williams KM, Ronaghy S. Use of simple measures of physical activity to predict stress fractures in young men undergoing a rigorous physical training program. Am J Epidemiol. 1999;149(3):236-42.

16. Shwayhat AF, Linenger JM, Hofherr LK, Slymen DJ, Johnson CW. Profiles of exercise history and overuse injuries among United States Navy Sea, Air, and Land (SEAL) recruits. Am J Sports Med. 1994;22(6):835-40.

17. Taanila H, Suni J, Pihlajamaki H, Mattila VM, Ohrankammen O, Vuorinen P, et al. Aetiology and risk factors of musculoskeletal disorders in physically active conscripts: a follow-up study in the Finnish Defence Forces. BMC Musculoskelet Disord. 2010;11:146

18. Heir T, Eide G. Injury proneness in infantry conscripts undergoing a physical training programme: smokeless tobacco use, higher age, and low levels of physical fitness are risk factors. Scand J Med Sci Sports. 1997;7(5):304-11.

19. Pope RP, Herbert RD, Kirwan JD, Graham BJ. A randomized trial of preexercise stretching for prevention of lower-limb injury. Med Sci Sports Exerc. 2000;32(2):271-7.
20. Blacker SD, Wilkinson DM, Bilzon JL, Rayson MP. Risk factors for training injuries among British Army recruits. Mil Med. 2008;173(3):278-86.

21. Grier TL, Morrison S, Knapik JJ, Canham-Chervak M, Jones BH. Risk factors for injuries in the U.S. Army Ordnance School. Mil Med. 2011;176(11):1292-9.

22. Knapik JJ, Sharp MA, Canham-Chervak M, Hauret K, Patton JF, Jones BH. Risk factors for training-related injuries among men and women in basic combat training. Med Sci Sports Exerc. 2001;33(6):946-54.

23. Jones BH, Knapik JJ. Physical training and exercise-related injuries. Surveillance, research and injury prevention in military populations. Sports Med. 1999;27(2):111-25.

24. Mattila VM, Parkkari J, Korpela H, Pihlajamaki H. Hospitalisation for injuries among Finnish conscripts in 1990-1999. Accid Anal Prev. 2006;38(1):99-104

25. Smith GS, Dannenberg AL, Amoroso PJ. Hospitalization due to injuries in the military. Evaluation of current data and recommendations on their use for injury prevention. Am J Prev Med. 2000;18(3 Suppl):41-53.

26. Songer TJ, LaPorte RE. Disabilities due to injury in the military. Am J Prev Med. 2000;18(3 Suppl):33-40.

27. Yancosek KE, Roy T, Erickson M. Rehabilitation programs for musculoskeletal injuries in military personnel. Curr Opin Rheumatol. 2012;24(2):232-6.

28. Sahi T, Korpela H: Varusmiespalveluksen keskeytyminen terveydellisistä syistä vuosina 1997-2000. [Interruptions in conscript service for health reasons in 1997-2000]. [In Finnish]. Sotilaslääketieteen laitoksen julkaisuja 2002(1):4-14

29. Taanila H, Hemminki AJ, Suni JH, Pihlajamaki H, Parkkari J. Low physical fitness is a strong predictor of health problems among young men: a follow-up study of 1411 male conscripts. BMC Public Health. 2011;11:590.

30. Santtila M, Tiainen S. Kuntotestaus Puolustusvoimissa. [Physical fitness tests in Finnish Defence Forces]. [In Finnish]. In: Keskinen KL, Häkkinen K, Kallinen M, editors. Kuntotestauksen Käsikirja. Helsinki: Liikuntatieteellinen Seura ry; 2004. p. 204-8.

31. Requa RK, Garrick JG. Adult Recreational Fitness. In: Caine DJ, Caine CG, Lindner KJ, editors. Epidemiology of Sport Injuries. Champaign, IL: Human Kinetics; 1996. p. 14-28.

32. Pasanen $K$, Parkkari J, Pasanen M, Hiilloskorpi H, Makinen $T$, Jarvinen $M$, et al. Neuromuscular training and the risk of leg injuries in female floorball players: cluster randomised controlled study. BMJ. 2008;337:a295.

33. Soligard T, Myklebust G, Steffen K, Holme I, Silvers H, Bizzini M, et al. Comprehensive warm-up programme to prevent injuries in young female footballers: cluster randomised controlled trial. BMJ. 2008;337:a2469.

34. Orava S. Exertion injuries due to sports and physical exercise. A clinical and statistical study of nontraumatic overuse injuries of the musculoskeletal system of athletes and keep-fit athletes, PhD thesis. Oulu, Finland: University of Oulu; 1980

35. Kleinbaum DG, Klein M. Survival analysis: a self-learning text. In: Statistics for biology and health. 3rd ed. New York: Springer Science + Business Media; 2012.

36. Bell NS, Mangione TW, Hemenway D, Amoroso PJ, Jones BH. High injury rates among female army trainees: a function of gender? Am J Prev Med. 2000;18(3 Suppl):141-6.

37. Garcin M, Mille-Hamard L, Billat V. Influence of aerobic fitness level on measured and estimated perceived exertion during exhausting runs. Int J Sports Med. 2004;25(4):270-7.

38. Willson JD, Kernozek TW. Plantar loading and cadence alterations with fatigue. Med Sci Sports Exerc. 1999;31(12):1828-33.

39. Benjaminse A, Habu A, Sell TC, Abt JP, Fu FH, Myers JB, et al. Fatigue alters lower extremity kinematics during a single-leg stop-jump task. Knee Surg Sports Traumatol Arthrosc. 2008;16(4):400-7.

40. Johnston 3rd RB, Howard ME, Cawley PW, Losse GM. Effect of lower extremity muscular fatigue on motor control performance. Med Sci Sports Exerc. 1998;30(12):1703-7.

41. Gleeson NP, Reilly T, Mercer TH, Rakowski S, Rees D. Influence of acute endurance activity on leg neuromuscular and musculoskeletal performance. Med Sci Sports Exerc. 1998;30(4):596-608.

42. Morris M, Dawes H, Howells K, Janssen R: Motor impairment and its relationship to fitness in children. BMJ open 2013, 3(7). doi:10.1136/ bmjopen-2013-002909.

43. McNeill W. Core stability is a subset of motor control. J Bodyw Mov Ther. 2010;14(1):80-3.

44. Leetun DT, Ireland ML, Willson JD, Ballantyne BT, Davis IM. Core stability measures as risk factors for lower extremity injury in athletes. Med Sci Sports Exerc. 2004;36(6):926-34. 
45. Ross J, Woodward A. Risk factors for injury during basic military training. Is there a social element to injury pathogenesis? J Occup Med. 1994;36(10):1120-6.

46. Rosendal L, Langberg H, Skov-Jensen A, Kjaer M. Incidence of injury and physical performance adaptations during military training. Clin J Sport Med. 2003;13(3):157-63.

47. Jones BH, Thacker SB, Gilchrist J, Kimsey Jr CD, Sosin DM. Prevention of lower extremity stress fractures in athletes and soldiers: a systematic review. Epidemiol Rev. 2002;24(2):228-47.

48. Jakobsson BT, Lundvall S, Redelius K, Engström L-M. Almost all start but who continue? A longitudinal study of youth participation in Swedish club sports. European Physical Education Review Sage Publications. 2012;18(1):3-18

49. Parkkari J, Taanila H, Suni J, Mattila VM, Ohrankammen O, Vuorinen P, et al. Neuromuscular training with injury prevention counselling to decrease the risk of acute musculoskeletal injury in young men during military service: a population-based, randomised study. BMC medicine. 2011;9:35

50. Suni JH, Taanila H, Mattila VM, Ohrankammen O, Vuorinen PC, Pihlajamaki $\mathrm{H}$, et al. Neuromuscular Exercise and Counseling Decrease Absenteeism Due to Low Back Pain in Young Conscripts: A Randomized. Population-Based Primary Prevention Study Spine. 2013;38(5):375-84.

51. Taanila H. Musculoskeletal disorders in male Finnish conscripts: Importance of physical fitness as a risk factor, and effectiveness of neuromuscular exercise and counseling in the prevention of acute injuries, and low back pain and disability, PhD Thesis. Tampere, Finland: University of Tampere; 2013.

52. Andersen LB, Wedderkopp N, Kristensen P, Moller NC, Froberg K, Cooper AR. Cycling to school and cardiovascular risk factors: a longitudinal study. J Phys Act Health. 2011;8(8):1025-33.

53. Yang X, Telama R, Hirvensalo M, Tammelin T, Viikari JS, Raitakari OT. Active commuting from youth to adulthood and as a predictor of physical activity in early midlife: the young Finns study. Prev Med. 2014;59:5-11.

54. Tammelin T, Laine K, Turpeinen S. Liikkuva koulu - ohjelman pilottivaiheen loppuraportti. [Final report on the Finnish Schools on the Move programme's pilot phase 2010-2012]. [In Finnish]. Jyväskylä, Finland: Liikunnan ja kansanterveyden edistämissäätiö LIKES; 2012.

55. Ekelund U, Tomkinson G, Armstrong N. What proportion of youth are physically active? Measurement issues, levels and recent time trends. Br J Sports Med. 2011;45(11):859-65.

56. Tammelin T, Aira A, Kulmala J, Kallio J, Kantomaa M, Valtonen M. Suomalaislasten fyysinen aktiivisuus - tavoitteena vähemmän istumista ja enemmän liikuntaa. [Physical activity among Finnish chlidren - less sedentary time and more physical activity needed]. [In Finnish]. Finnish Medical Journal. 2014;69:1871-6

57. Ortega FB, Konstabel K, Pasquali E, Ruiz JR, Hurtig-Wennlof A, Maestu J, et al. Objectively measured physical activity and sedentary time during childhood adolescence and young adulthood: a cohort study. PloS one. 2013;8(4):e60871.

58. Kolu P, Luoto R, Vasankari T. Liikkumattomuus ja terveydenhuollon kustannukset. [Physical inactivity and health care costs]. [In Finnish]. Finnish Medical Journal. 2014;69:885-9.

59. Wang G, Macera CA, Scudder-Soucie B, Schmid T, Pratt M, Buchner D. A cost-benefit analysis of physical activity using bike/pedestrian trails. Health Promot Pract. 2005:6(2):174-9.

60. Sevick MA, Napolitano MA, Papandonatos GD, Gordon AJ, Reiser LM, Marcus $\mathrm{BH}$. Cost-effectiveness of alternative approaches for motivating activity in sedentary adults: results of Project STRIDE. Prev Med. 2007;45(1):54-61.

61. Jones BH, Bovee MW, Harris 3rd JM, Cowan DN. Intrinsic risk factors for exercise-related injuries among male and female army trainees. Am J Sports Med. 1993;21(5):705-10.

62. Beck TJ, Ruff CB, Mourtada FA, Shaffer RA, Maxwell-Williams K, Kao GL, et al. Dual-energy $\mathrm{X}$-ray absorptiometry derived structural geometry for stress fracture prediction in male U.S. Marine Corps recruits. J Bone Miner Res. 1996;11(5):645-53

63. Knapik JJ, Reynolds KL, Harman E. Soldier load carriage: historical, physiological, biomechanical, and medical aspects. Mil Med. 2004;169(1):45-56

64. Vanderburgh PM. Occupational relevance and body mass bias in military physical fitness tests. Med Sci Sports Exerc. 2008;40(8):1538-45.
65. Mattila VM, Kuronen P, Pihlajamaki H. Nature and risk factors of injury hospitalization in young adults: a follow-up of 135,987 military conscripts. Scand J Public Health. 2007;35(4):418-23.

66. Peake J, Gargett S, Waller M, McLaughlin R, Cosgrove T, Wittert G, et al. The health and cost implications of high body mass index in Australian defence force personnel. BMC Public Health. 2012;12(1):451.

67. Santtila M, Kyrolainen H, Vasankari T, Tiainen S, Palvalin K, Hakkinen A, et al. Physical fitness profiles in young Finnish men during the years 1975-2004. Med Sci Sports Exerc. 2006;38(11):1990-4.

68. Ferraro KF, Booth TL. Age, body mass index, and functional illness. J Gerontol B Psychol Sci Soc Sci. 1999;54(6):S339-48.

69. Lakdawalla DN, Bhattacharya J, Goldman DP. Are the young becoming more disabled? Health affairs. 2004;23(1):168-76.

70. Larsson UE, Mattsson E. Perceived disability and observed functional limitations in obese women. Int J Obes Relat Metab Disord. 2001;25(11):1705-12.

71. Hue O, Simoneau M, Marcotte J, Berrigan F, Dore J, Marceau P, et al. Body weight is a strong predictor of postural stability. Gait \& posture. 2007;26(1):32-8.

72. Rodacki AL, Fowler NE, Provensi CL, Rodacki Cde L, Dezan VH. Body mass as a factor in stature change. Clin Biomech (Bristol, Avon). 2005;20(8):799-805.

73. Reynolds K, Cosio-Lima L, Creedon J, Gregg R, Zigmont T. Injury occurrence and risk factors in construction engineers and combat artillery soldiers. Mil Med. 2002;167(12):971-7.

74. Teasdale N, Hue O, Marcotte J, Berrigan F, Simoneau M, Dore J, et al. Reducing weight increases postural stability in obese and morbid obese men. Int J Obes (Lond). 2007;31(1):153-60.

75. Santtila M, Keijo H, Laura K, Heikki K. Changes in cardiovascular performance during an 8-week military basic training period combined with added endurance or strength training. Mil Med. 2008;173(12):1173-9.

76. Absetz $P$, Jallinoja $P$, Suihko J, Bingham C, Kinnunen M, Ohrankämmen $\mathrm{O}$, et al. Varusmiesten ravitsemus ja muut elintavat sekä terveyden riskitekijät palveluksen aikana. Kuuden kuukauden seurantatutkimus, [Nutrition and other lifestyle habits as well as health risk factors of conscripts during military service. A six-month follow-up study]. [In Finnish]. Report 16/2010. Helsinki, Finland: National Institute for Health and Welfare (THL); 2010.

77. Lehesjoki M, Parkkola K, Peitso A. Sotilaan kenttäkelpoisuuden arvionti [Judgement of conscript's combat ability]. [In Finnish]. Sotilaslääketieteellinen Aikakauslehti [Annales Medicinae Militrais Fenniae]. 2010;1:16-9.

78. Mattila VM, Tallroth K, Marttinen M, Pihlajamaki H. Physical fitness and performance. Body composition by DEXA and its association with physical fitness in 140 conscripts. Med Sci Sports Exerc. 2007;39(12):2242-7.

79. Olsen OE, Myklebust G, Engebretsen L, Holme I, Bahr R. Exercises to prevent lower limb injuries in youth sports: cluster randomised controlled trial. BMJ. 2005;330(7489):449.

80. Walden M, Atroshi I, Magnusson H, Wagner P, Hagglund M. Prevention of acute knee injuries in adolescent female football players: cluster randomised controlled trial. BMJ. 2012;344:e3042

81. Knapik JJ, Darakjy S, Hauret KG, Canada S, Scott S, Rieger W, et al. Increasing the physical fitness of low-fit recruits before basic combat training: an evaluation of fitness, injuries, and training outcomes. Mi Med. 2006;171(1):45-54.

82. Knapik JJ, Bullock SH, Canada S, Toney E, Wells JD, Hoedebecke E, et al. Influence of an injury reduction program on injury and fitness outcomes among soldiers. Inj Prev. 2004;10(1):37-42.

83. Finestone A, Milgrom C. How stress fracture incidence was lowered in the Israeli army: a 25-yr struggle. Med Sci Sports Exerc. 2008;40(11 Suppl):S623-9.

84. Coppack RJ, Etherington J, Wills AK. The effects of exercise for the prevention of overuse anterior knee pain: a randomized controlled trial. Am J Sports Med. 2011;39(5):940-8.

85. Brushoj C, Larsen K, Albrecht-Beste E, Nielsen MB, Loye F, Holmich P. Prevention of overuse injuries by a concurrent exercise program in subjects exposed to an increase in training load: a randomized controlled trial of 1020 army recruits. Am J Sports Med. 2008;36(4):663-70.

86. Salo M. Determinants of military adjustment and attrition during Finnish conscript service. Publication series 2. Research report No 21, PhD thesis. Tampere, Finland: University of Tampere; 2008. 